\title{
Binary neutron star mergers: Effects of spin and post-merger dynamics
}

\author{
William E. East, ${ }^{1}$ Vasileios Paschalidis, ${ }^{2}$ Frans Pretorius, ${ }^{3,4}$ and Antonios Tsokaros ${ }^{5}$ \\ ${ }^{1}$ Perimeter Institute for Theoretical Physics, Waterloo, Ontario N2L 2Y5, Canada \\ ${ }^{2}$ Departments of Astronomy and Physics, University of Arizona, Tucson, Arizona 85721, USA \\ ${ }^{3}$ Department of Physics, Princeton University, Princeton, New Jersey 08544, USA \\ ${ }^{4}$ Canadian Institute for Advanced Research, Cosmology \& Gravity Program, \\ Toronto, Ontario M5G 1Z8, Canada \\ ${ }^{5}$ Department of Physics, University of Illinois at Urbana-Champaign, Urbana, Illinois 61801, USA
}

(Received 19 June 2019; published 17 December 2019)

\begin{abstract}
Spin can have significant effects on the electromagnetic transients accompanying binary neutron star mergers. The measurement of spin can provide important information about binary formation channels. In the absence of a strong neutron star spin prior, the degeneracy of spin with other parameters leads to significant uncertainties in their estimation, in particular limiting the power of gravitational waves to place tight constraints on the nuclear equation of state. Thus detailed studies of highly spinning neutron star mergers are essential to understand all aspects of multimessenger observation of such events. We perform a systematic investigation of the impact of neutron star spin-considering dimensionless spin values up to $a_{\mathrm{NS}}=0.33$ - on the merger of equal mass, quasicircular binary neutron stars using fully general-relativistic simulations. We find that the peak frequency of the post-merger gravitational wave signal is only weakly influenced by the neutron star spin, with cases where the spin is aligned (antialigned) with the orbital angular momentum giving slightly lower (higher) values compared to the irrotational case. We find that the one-arm instability arises in a number of cases, with some dependence on spin. Spin has a pronounced impact on the mass, velocity, and angular distribution of the dynamical ejecta, and the mass of the disk that remains outside the merger remnant. We discuss the implications of these findings on anticipated electromagnetic signals, and on constraints that have been placed on the equation of state based on multimessenger observations of GW170817.
\end{abstract}

DOI: $10.1103 /$ PhysRevD.100.124042

\section{INTRODUCTION}

During the first two observing runs (O1 and $\mathrm{O} 2)$, the LIGO and Virgo Scientific Collaborations detected a number of gravitational wave (GW) signals consistent with the inspiral and merger of binary black holes [1-6], and one GW signal consistent with the inspiral of a binary neutron star (NS) [7] (GW170817). GW170817 was accompanied by a number of observed electromagnetic counterparts [8], including a short gamma-ray burst [9], and an ultraviolet/optical/near-infrared transient consistent with the radioactive decay of heavy elements formed in rapidly expanding neutron-rich matter, i.e., a kilonova [8]. These breakthroughs not only opened a new window for observing our Universe, but also hold promise that during the third observing run we could have hundreds of new events [1]. Multimessenger observations are key to solving some longstanding puzzles in fundamental physics and astrophysics. For example, observations from GW170817 have already been used to place new constraints on the behavior of matter at supernuclear densities (see e.g., [7,10-21], and also [22,23] for reviews), and the sites where heavy elements in the Universe form (see, e.g., [24] and references therein). GW170817 has also been used to independently measure the Hubble constant [25], and to constrain the nature of gravity [7]. GW170817 ruled out a large class of modified gravity theories [26-32] by placing a stringent limit on the difference in propagation speed of GW and electromagnetic waves [28]. The wealth of physics extracted from these first observations has been unprecedented. The fact that the total observation time of $\mathrm{O} 1$ and O2 was only about one year (and not all detectors were online at all times) and the fact that the third observing run (O3) will have increased sensitivity, promise that during $\mathrm{O} 3$ there will be many more additional events and-hopefully—new surprises.

The LIGO/Virgo observation of GW170817 also gave rise to new questions and puzzles. A particular aspect of the GW170817 observation that is of interest to this work is that only very weak constraints were placed on the premerger NS spins. Thus, an important question regarding GW170817 is the following: what was the pre-merger rotation state of the NSs involved in the event? This is important for a number of reasons. To begin with, uncertainty in the NS spins prior to merger leads to large 
uncertainties in other inferred binary parameters, such as the binary NS total mass and mass ratio, and the tidal deformability, because of degeneracies in how these parameters affect the GW signal [7]. Most studies placing constraints on the NS equation of state (EOS) assumed the results from the low-spin prior LIGO analysis of GW170817. While this assumption is motivated by the fact that the stars in Galactic double NSs are observed to spin slowly, it nevertheless leads to inferences about other aspects of the system (including constraints on the nuclear EOS) that may be biased by our prior observations. On the other hand, the observation of non-negligible spin in a merging binary NS would automatically give revealing information about the formation channel that gave rise to the binary, perhaps pointing to a subpopulation of dynamically assembled binaries [33].

Ideally, we want to be able to use these new observations to test our assumptions and make independent measurements by finding ways orthogonal to the inspiral GW signal to constrain the NS spins involved in these mergers. For example, NS spin can potentially affect the lifetime of the merger remnant before it collapses to a black hole, the amount of disk mass forming outside the remnant, and/or the mass in dynamical ejecta, all of which affect kilonova signatures and potential gamma-ray (jet) signatures (see, e.g., [34] for a recent review). Thus, combining electromagnetic observations and detailed theoretical modeling of binary NS mergers that accounts for NS spin with GW observations has the potential for more accurate parameter inference.

Dynamical spacetime simulations of spinning binary NSs are necessary to address these issues. Through these simulations one can compute the impact of spin on both the GW and electromagnetic signatures. Simulations of spinning binary NSs are currently under way by several groups, see, e.g., [35-42] for quasicircular mergers with constraintsatisfying, quasiequilibrium initial data, and [43-45] where the initial data are constraint violating and not in equilibrium. In [46] simulations were also presented in the conformal flatness approximation of general relativity. Finally, we performed studies of eccentric binaries with spinning NSs, employing constraint-satisfying initial data in [33,47-49].

Here, we present results from fully relativistic hydrodynamic simulations of quasiequilibrium binary NSs in quasicircular orbits with spinning components. The binary configurations are of equal mass and equal spin, with the spin vectors either aligned or antialigned with the orbital angular momentum. The initial dimensionless NS spins are in the range $a_{\mathrm{NS}} \in[-0.13,0.33]$, where a positive (negative) sign denotes the corresponding vectors are aligned (antialigned) with the orbital angular momentum. The matter is modeled with different EOSs which are represented as piecewise polytropes and cover a range of compactness for a $1.4 M_{\odot}$ NS from $\sim 0.136$ to 0.178 . We study how spin affects the dynamics of the merger, the post-merger GW signals, dynamical ejecta, and the merger remnant disk mass. We also include several nonspinning cases with different EOSs to illustrate how these effects can be degenerate with varying EOS, and in order to compare to previous studies.

Our simulations demonstrate that the post-merger peak GW frequency is only weakly influenced by the NS spin (by about 100-200 Hz). This is consistent with the results of Refs. [35,37] as well as Ref. [46], the latter using the conformal flatness approximation to general relativity. We find that aligned (antialigned) spin cases give slightly lower (higher) values of the post-merger peak GW frequency when compared to the irrotational case. In turn, this implies that there is some degree of degeneracy with the nuclear EOS when inferring the latter from the post-merger peak GW frequency. We find that the one-arm instability ${ }^{1}$ we discovered in eccentric NS mergers (including those with spin) [47-49] and studied in select nonspinning quasicircular mergers [50,51], also operates in quasicircular mergers with spin, though the correlation between the strength of the one-arm mode and the pre-merger spin magnitude is not strong. In particular, we find that the strongest one-arm mode develops for an intermediate value of NS spin that we consider. The GWs from post-merger NS oscillations could potentially be detected by alternative configurations of current observatories optimized for kilohertz frequencies, as well as third-generation GW detectors [52].

We demonstrate that spin has a substantial impact on the mass, velocity and angular distribution of dynamical ejecta, and the subsequent red kilonova signatures. Our results indicate that spins antialigned with the orbital angular momentum result in more massive dynamical ejecta, with a non-negligible amount of matter traveling at speeds near $0.5 \mathrm{c}$. As a result, our study suggests that the radio signatures of antialigned binary NS (BNS) mergers are expected to be significantly brighter. Moreover, our results suggest that antialigned spin mergers generate brighter red kilonovae than aligned-spin cases, which have smaller dynamical ejecta masses. However, we find that as the aligned spin increases past a certain value, the amount of dynamical ejecta increases again. This implies that the expected red kilonovae should become brighter for higher spin valuesconsistent with the fact that as the spin frequency increases, the star becomes less bound, and hence becomes easier to dynamically eject more mass. For larger aligned spins, our results show that the dynamical ejecta are more concentrated near the orbital plane. Importantly, for dimensionless spins of order $0.2-0.3$, our calculations show that merger remnants have larger disks than lower-spin cases. Hence, blue kilonovae from such systems are likely to be brighter as the spin magnitude increases. The fact that as the spin magnitude increases we obtain heavier disks, implies that

\footnotetext{
${ }^{1}$ We note that here and throughout by "one-arm instability" we imply the existence of a "one-arm" $(m=1)$ mode that grows out of tiny perturbations.
} 
the recent constraints on the binary tidal deformability discussed in [16] may be even weaker than found in [53], especially since the GW analysis of GW170718 finds that the $90 \%$ confidence interval for the NS dimensionless spins in GW170817 extends up to 0.6 [54]. Similar constraints placed on the EOS may need to be revisited, because related works do not consider the impact of pre-merger spin.

Finally, we compare two simulations that have the same initial properties, i.e., same total mass, orbital angular frequency, and equatorial circulation, but one corresponds to corotation and the other to the corresponding configuration built with the constant rotational-velocity formulation [55-57]. We find some differences in the post-merger evolution of the two configurations, but they are broadly consistent with each other. In particular, the similarity of the GW emission implies that the bulk of the matter profiles are quite similar between the two formulations. Though we do see rather different (but in both cases relativity small) ejecta masses following merger, this may imply the outer layers in the stars have slightly different properties between the two formulations. Given the definition of circulation, and that the velocity decomposition used in the constant rotational-velocity formulation is ad hoc, this is expected (see discussion in the Appendix of Ref. [57]).

The remainder of this paper is structured as follows. In Sec. II, we briefly describe the numerical methods and codes we use, including the initial data and the EOSs we treat in this work. In Sec. III, we present the results from our numerical simulations and discuss their astrophysical implications. We conclude in Sec. IV with a summary of our main findings and a discussion of their implications. In Appendix A, we include details of the comparison of corotation versus constant rotational-velocity methods of construction spinning binary NSs, and in Appendix B we give some details on numerical convergence. Unless otherwise stated, we use geometrized units with $G=c=1$.

\section{NUMERICAL APPROACH}

We simulate BNS mergers by evolving the Einstein equations coupled to hydrodynamics using the code described in Ref. [58]. We discretize the Einstein field equations in the generalized-harmonic formulation using fourth-order accurate finite differences and time integration. We model the NS matter as a perfect fluid, and evolve the general-relativistic Euler equations in conservative form using the specific high-resolution shock-capturing techniques detailed in Ref. [59].

\section{A. Initial conditions}

Our initial data correspond to unmagnetized, quasiequilibrium BNSs in a quasicircular orbit. For this study, we restrict to binaries consisting of two identical NSs, modeled by piecewise polytropic EOSs. We consider cases where each binary companion has an initial quasilocal dimensionless spin of $a_{\mathrm{NS}}:=J_{\mathrm{ql}} /(M / 2)^{2}=-0.13,0.08$, $0.17,0.25$, and 0.33 , where $J_{\mathrm{ql}}$ is the quasilocal angular momentum of the NS, and $M$ is the Arnowitt-Deser-Misner (ADM) mass of the binary. For the definition of the quasilocal angular momentum and subtleties related with it see [57]. More specifically, we do not fix the dimensionless spin, but the circulation, which here is in the range of $-0.7 \mathcal{C}_{\text {cor }}$ to $1.9 \mathcal{C}_{\text {cor }}$, where $\mathcal{C}_{\text {cor }}$ is the circulation of the corotating binary at that separation [57]. The spinning configurations are built with the constant rotationalvelocity formulation of Refs. [55,56]. Among the spinning configurations we include two cases that have the same circulation, ADM mass, and angular velocity, but one is constructed with the corotation formalism described in Ref. [60], and the other with the constant rotationalvelocity formalism. The dimensionless spin in both cases is $a_{\mathrm{NS}}=0.17$. Given that the two $a_{\mathrm{NS}}=0.17$ configurations are built with different formulations, it is not a priori clear that they describe the same physical system. Therefore, such comparisons can serve to elucidate the physics of the constant rotational-velocity formalism for BNS initial data in a well-understood regime. The details of the comparison of the simulations with these two configurations are discussed in Appendix A. All initial data are computed using the Compact Object CALculator [57,61], and their main properties are listed in Table I. The residual eccentricity for these initial data is $\sim 0.005$ [42]. For most cases (including all those with spinning NSs) we use the piecewise polytropic representation of the ENG EOS [62] from [63]. We also study several nonspinning (irrotational) configurations using the ENG, $2 \mathrm{H}, \mathrm{H}$, and $\mathrm{HB}$ EOSs from [64]. These cases are included to give a comparison as to the degree to which the effects of varying spin are similar to varying EOS, and in particular to be comparable to the study of eccentric mergers performed in Ref. [49]. The radius of a $1.35 M_{\odot}$ nonspinning NS with these equations of state is in the range $[11.6,15.2] \mathrm{km}$. For the evolutions, we add an additional thermal component to the pressure $P_{\text {th }}=$ $0.5 \rho_{0} \epsilon_{\text {hot }}$ (motivated by [65]) where $\epsilon_{\text {hot }}$ is the specific energy in excess of that prescribed by the cold EOS.

\section{B. Diagnostics}

In order to analyze the simulations, we use several diagnostic quantities. We extract the gravitational radiation by evaluating the Newman-Penrose scalar $\psi_{4}$ in the wave zone. We decompose this quantity on spheres at large radii (typically $r=100 M$ ) into spin -2 weighted spherical harmonics with coefficients $C_{\ell m}$. We also give a frequency domain representation of the GWs by computing the characteristic strain $h_{c}=|\tilde{h}| f$ in terms of the Fourier transform of the strain $\tilde{h}$ and the frequency $f$.

To characterize the post-merger ejected matter, we use the integrated rest-mass density $\rho_{0}$ residing outside some given radius 
TABLE I. Properties of the initial BNS configurations. Listed are the EOS, the binary ADM mass $M$, the dimensionless ADM angular momentum $J / M^{2}$, the NS quasilocal dimensionless spin parameter $a_{\mathrm{NS}} \equiv J_{\mathrm{ql}} /(M / 2)^{2}$ (aligned “+” or antialigned "-" with orbital angular momentum), the approximate rotational period $T$ [57], ratio of the coordinate equatorial radius toward companion $R_{x}$ to the coordinate polar radius $R_{z}$, the orbital separation is $D$, corresponding to an initial binary angular velocity of $M \Omega$, and circulation $\mathcal{C}$. The last column indicates if the binary is a spinning "SP," irrotational "IR" or corotating "CO" configuration.

\begin{tabular}{lccccccccc}
\hline \hline EOS & $M\left[\mathrm{M}_{\odot}\right]$ & $J / M^{2}$ & $a_{\mathrm{NS}}$ & $T[\mathrm{~ms}]$ & $R_{z} / R_{x}$ & $D[\mathrm{~km}]$ & $M \Omega$ & $C\left[\mathrm{M}_{\odot}\right]$ & Spin state \\
\hline ENG & 2.72 & 0.93 & -0.13 & 4.12 & 0.96 & 41.85 & 0.026 & -3.00 & SP \\
ENG & 2.72 & 0.99 & 0.00 & N/A & 0.97 & 41.80 & 0.026 & 0.00 & IR \\
ENG & 2.72 & 1.02 & 0.08 & 6.14 & 0.97 & 41.77 & 0.026 & 2.00 & SP \\
ENG & 2.72 & 1.07 & 0.17 & 3.01 & 0.95 & 41.80 & 0.026 & 4.15 & SP \\
ENG & 2.72 & 1.06 & 0.17 & 3.23 & 0.95 & 41.67 & 0.026 & 4.15 & CO \\
ENG & 2.72 & 1.11 & 0.25 & 2.13 & 0.92 & 41.89 & 0.026 & 6.00 & SP \\
ENG & 2.72 & 1.15 & 0.33 & 1.65 & 0.89 & 42.04 & 0.026 & 8.00 & SP \\
2H & 2.70 & 1.03 & 0.00 & N/A & 0.94 & 46.66 & 0.022 & 0.00 & IR \\
H & 2.70 & 0.96 & 0.00 & N/A & 0.95 & 38.09 & 0.030 & 0.00 & IR \\
HB & 2.70 & 0.99 & 0.00 & N/A & 0.97 & 41.62 & 0.026 & 0.00 & IR \\
\hline \hline
\end{tabular}

$$
M_{0}(>r)=\int_{>r} \rho_{0} u^{t} \sqrt{-g} d^{3} x
$$

where $u^{t}$ is the $t$ component of the fluid 4-velocity. Postmerger, part of the rest mass will become unbound and escape to infinity. We use the criteria that $u_{t}<-1$ and the radial component of the velocity be positive in flagging fluid elements as unbound. From the value of $u_{t}$, we can also determine the distribution of the rest mass $M_{0}$ over values of the velocity at infinity $v_{\infty}$.

For comparison, we give a rough estimate of how these measured properties of the ejecta might translate into observable astrophysical transients. We do this by use of calculations of such processes that suggest a rise time for kilonovae light curves of [66]

$$
t_{\text {peak }} \approx 0.3\left(\frac{M_{0, \mathrm{u}}}{10^{-2} M_{\odot}}\right)^{1 / 2}\left(\frac{v}{0.2 c}\right)^{-1 / 2} \mathrm{~d},
$$

measured from the merger, and peak luminosities of

$$
L \approx 1.6 \times 10^{41}\left(\frac{M_{0, \mathrm{u}}}{10^{-2} M_{\odot}}\right)^{1 / 2}\left(\frac{v}{0.2 c}\right)^{1 / 2} \operatorname{erg} s^{-1} .
$$

Here, $M_{0, \mathrm{u}}$ and $v$ are the rest mass and characteristic velocity of the unbound ejecta. Note that Eqs. (2) and (3) depend on the opacity of the ejecta, which depends on their composition. Here we scale the equations such that they are in agreement with the results of [66]. It is possible that ejecta from mergers with spinning neutron stars and different equations of state have slightly different composition than nonspinning ones, thus Eqs. (2) and (3) are used to provide simple estimates and to understand any trends. Detailed radiative transfer calculations of ejected matter are necessary for robust kilonova calculations, see e.g., [67].

Typical unbound ejecta masses from BNS mergers are of order $10^{-3}-10^{-2} M_{\odot}$. However, the observed kilonova accompanying GW170817 has been explained by invoking ejecta masses of order $0.025-0.05 M_{\odot}$ [24,68-77], and hence significantly larger than the dynamical ejecta masses. Moreover, the kilonova associated with GW170817 seems to require at least two components to explain the observed color evolution: one component accounting for the red kilonova (explained by highopacity, lanthanide-rich outflows associated with lowelectron fraction dynamical ejecta), and one component accounting for the blue kilonova (explained by lowopacity, lanthanide-poor outflows associated with highelectron fraction disk wind material). Therefore, it is not likely that dynamical ejecta from BNSs can, by itself, explain such bright electromagnetic signatures.

Recent studies [78-84] suggest that a significant fraction of the mass of the disk that forms around the BNS merger remnant becomes unbound because of viscous, neutrino, and magnetic field processes. Thus, a disk mass of order 0.05-0.1 $M_{\odot}$ can explain the kilonova that accompanied GW170817. For these reasons, we also estimate the disk rest mass as the bound mass outside the remnant black hole. In those cases where a longer-lived massive NS forms following merger, we define the disk rest mass as the bound rest mass outside a radius of $\approx 30 \mathrm{~km}$, which is where, in our simulations, the rest-mass density roughly drops by 3 orders of magnitude from the maximum rest-mass density of the massive NS remnant. We point out that the definition of disk mass is not unambiguous (see e.g., [85]). However, if we define the remnant star surface as the isosurface with rest-mass density 2 orders of magnitude smaller than the maximum value of the rest-mass density, then the stellar radii in all of our simulations are all smaller than $20 \mathrm{~km}$. In this sense, the disk masses we compute for radii $>30 \mathrm{~km}$ are a lower limit.

Another electromagnetic transient associated with material ejected in compact object mergers is radio emission when this material sweeps the interstellar medium [86]. These signals typically peak on time scales [86] 
$t_{F} \approx 6\left(\frac{E_{\mathrm{kin}}}{10^{51 \mathrm{erg}}}\right)^{1 / 3}\left(\frac{n_{0}}{0.1 \mathrm{~cm}^{-3}}\right)^{-1 / 3}\left(\frac{v}{0.3 c}\right)^{-5 / 3} \mathrm{yr}$

with brightness

$$
\begin{aligned}
F\left(\nu_{\mathrm{obs}}\right) \approx & 0.6\left(\frac{E_{\mathrm{kin}}}{10^{51} \mathrm{erg}}\right)\left(\frac{n_{0}}{0.1 \mathrm{~cm}^{-3}}\right)^{7 / 8} \\
& \times\left(\frac{v}{0.3 c}\right)^{11 / 4}\left(\frac{\nu_{\mathrm{obs}}}{\mathrm{GHz}}\right)^{-3 / 4}\left(\frac{d}{100 \mathrm{Mpc}}\right)^{-2} \mathrm{mJy} .
\end{aligned}
$$

In the equations above, $E_{\text {kin }}$ is the kinetic energy of the ejecta, $\nu_{\text {obs }}$ is the observation frequency, $d$ the distance to source, and we use a fiducial value of $n_{0} \sim 0.1 \mathrm{~cm}^{-3}$ for the interstellar medium density.

In cases where a massive NS remnant forms post-merger, we also characterize the matter using several quantities. We decompose the density distribution into different azimuthal modes

$$
C_{m}=\int \rho_{0} u^{t} \sqrt{-g} e^{i m \phi} d^{3} x
$$

where $\phi$ is the coordinate azimuthal angle and $m$ is an integer, which is useful for monitoring the onset of shear/ nonaxisymmetric instabilities. In particular, for all the configurations considered here, $C_{m}$ is initially zero for odd $m$, and becomes nonzero through such instabilities.

For these massive NS remnants, we also examine the rotational profile of the post-merger star using an azimuthal average of the angular velocity $\Omega=u^{\phi} / u^{t}$ over fixed values of the cylindrical coordinate radius $\varpi=\sqrt{x^{2}+y^{2}}$, where we compute $u^{\phi}$, and $\varpi$ in the initial center-of-mass frame of the binary, i.e., the origin of the coordinate system.

\section{Resolution}

For all simulations, we utilize six levels of adaptive mesh refinement each with 2:1 refinement ratio. For most of the results presented here, we use resolution with $193^{3}$ points on the base level, a resolution of $d x \approx 0.05 M \approx 0.2 \mathrm{~km}$ on the finest level, and a resolution of $d x \approx 1.6 M \approx$ $1 /(47 \mathrm{kHz})$ in the $\mathrm{GW}$ extraction zone. The grid structure is dynamically adjusted during the evolution based on truncation error estimates for the metric functions, while ensuring that the finest level always covers the star(s). See Ref. [58] for more details. To establish convergence, and estimate truncation errors, we also run two cases at $4 / 3 \times$ and $2 \times$ our canonical resolution. Results of the convergence study are presented in Appendix B.

\section{RESULTS AND DISCUSSION}

With the initial parameters used here, the BNSs undergo 4-6 orbits before merging. Of the mergers we study here, we find that - with the exception of the one case using the HB EOS - all produce a long-lived massive NS remnant that does not collapse on the timescales of our simulations (up to $\sim 30 \mathrm{~ms}$ post-merger). In the following, we characterize the gravitational wave signals, post-merger remnants, and unbound material from these cases with different EOSs and spins.

\section{A. Gravitational waves}

For the mergers of nonspinning NSs with different EOS, the post-merger GW amplitude is larger for softer EOSs that give rise to more compact NSs. This is illustrated in Fig. 1 which shows the dominant $\ell=m=2$ component of $\psi_{4}$. For the softest EOS considered, the HB, the BNS remnant collapses after $\sim 10 \mathrm{~ms}$ producing a black hole.

We also show the GWs for the ENG EOS and various values of NS spin in Fig. 2. We observe that higher prograde spin $\left(a_{\mathrm{NS}} \gtrsim 0.17\right)$ results in smoother post-merger GWs that do not exhibit "beat" oscillations as in the lowerspin cases. Moreover, the post-merger GW amplitude in nonzero spin cases decays more slowly in the first $20 \mathrm{~ms}$ than in the irrotational case, implying a stronger postmerger GW signal when spin is considered. This result is further supported by our resolution study in Appendix B.

The post-merger GW signal is strongly peaked in a narrow frequency range, as more clearly illustrated in the frequency domain decompositions shown in Figs. 3 and 4. Figure 3 shows how the peak frequency noticeably shifts to lower frequencies as stiffer EOSs are considered for irrotational binaries. The peak frequency is also weakly influenced by the NS spin (by about 100-200 Hz), as evident in Fig. 4, with the aligned (antialigned) spin cases giving slightly lower (higher) values compared to the

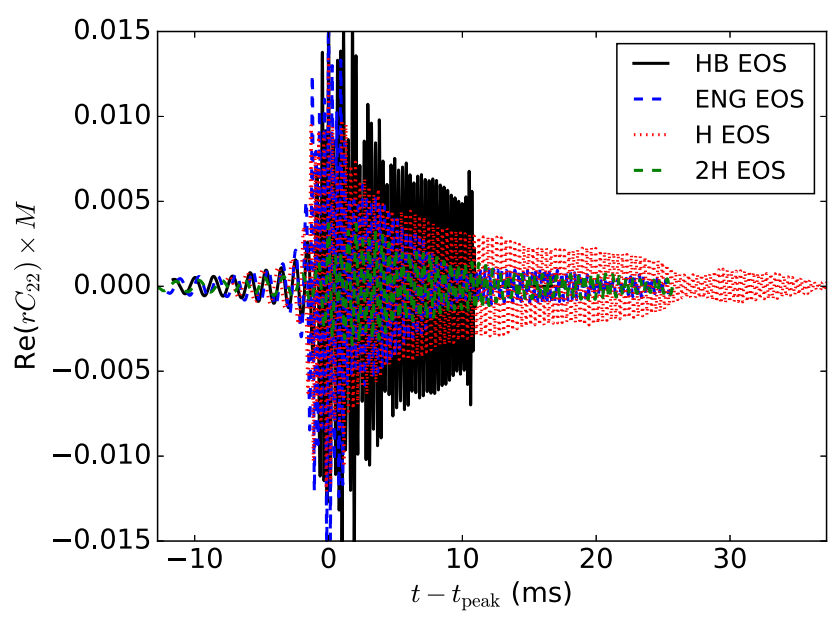

FIG. 1. The GW signal $\left(\ell=m=2\right.$ component of $\psi_{4}$ multiplied by the extraction radius $r$ ) from nonspinning mergers with various EOSs. The curves have been aligned in time and phase at peak. The HB EOS curve ends once a black hole $(\mathrm{BH})$ forms; the other cases did not collapse to BHs during the time of their respective simulations. 

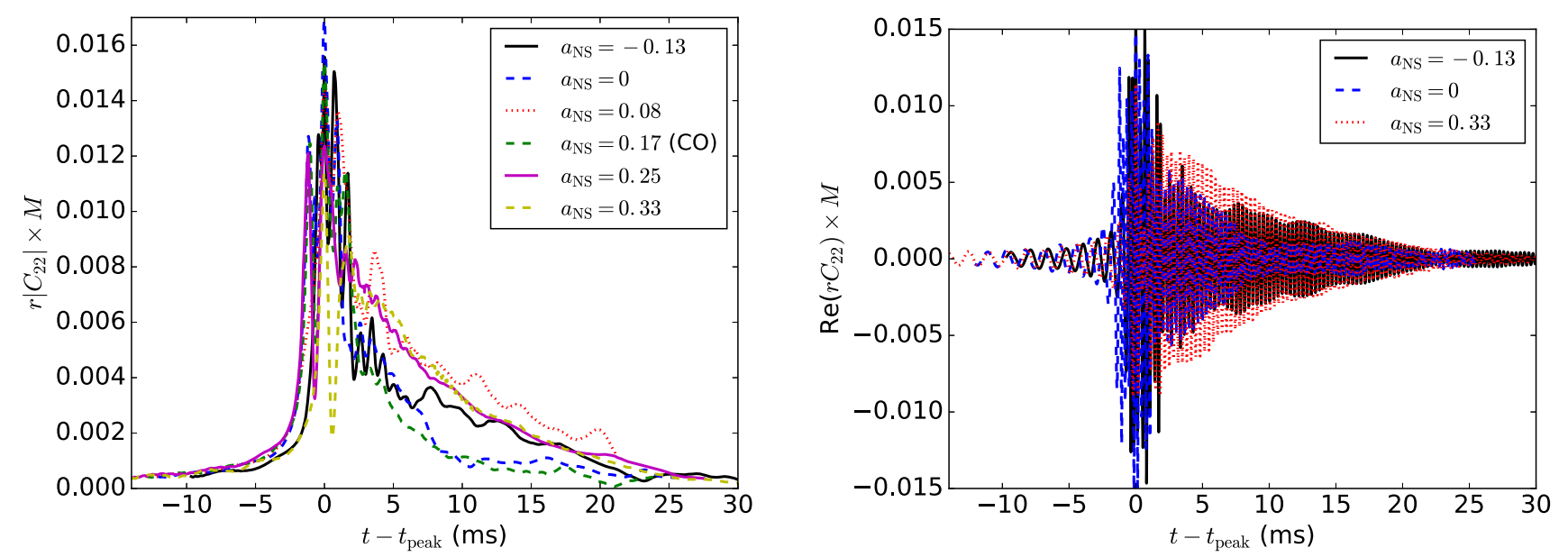

FIG. 2. The GW signal ( $\ell=m=2$ component of $\psi_{4}$ multiplied by the extraction radius $r$ ) from mergers with the ENG EOS and various values of NS spin. The left panel shows the magnitude of $\psi_{4}$, while the right panel shows the real part of $\psi_{4}$ for select cases. The curves have been aligned in time and (for the right panel) phase at peak. None of these cases collapsed to BHs during the time of their respective simulations.

nonspinning case. This implies that when considering the post-merger peak GW frequency as a means for constraining the nuclear EOS, there will be some degeneracy between the EOS and the spin. As noted above, the cases with higher spin magnitude also have somewhat more power at the peak frequency. There are also smaller components in the GW signal at other frequencies, as can be seen in Fig. 2.

From the left panel of Fig. 4, it is evident that the antialigned case has the most GW power in the higher frequency subdominant peaks, and that this power decreases strongly as the aligned NS spin is increased, though the frequency at which the peaks occur is relatively insensitive to the spin. However, such features at very high frequencies ( $\gtrsim 4 \mathrm{kHz}$ ) will be difficult to observe in the near future. At lower frequencies, a prominent feature in the edge-on view (right panel of Fig. 4) is the peak close to $1.6 \mathrm{kHz}$, which corresponds to the frequency of the onearm mode as found in studies of eccentric BNS mergers [47-49] and quasicircular mergers [50,51]. We discuss the post-merger dynamics of the one-arm mode in more detail in the following section, and here we focus on its detectability in the GW spectrum.

As pointed out in [47-49], the frequency of the one-arm mode occurs at half the peak frequency, and hence where LIGO/Virgo are more sensitive. Moreover, the massive NSs formed after merger emit almost monochromatic GWs at the one-arm mode frequency. We conjecture that this
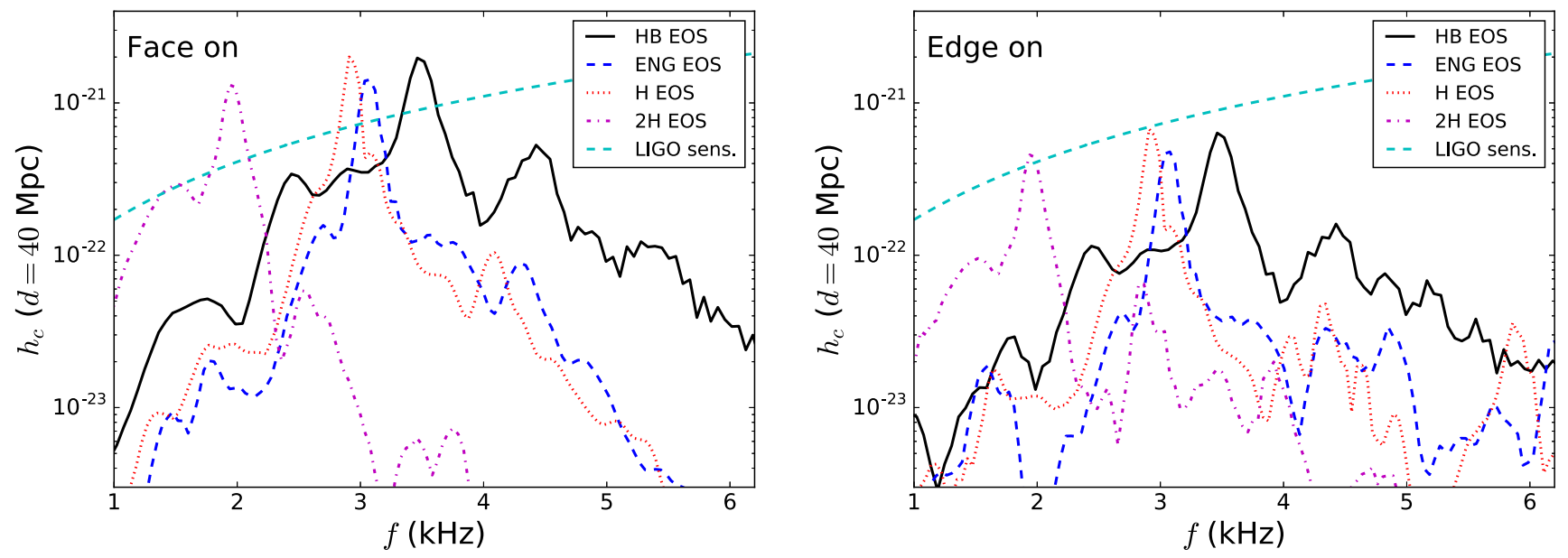

FIG. 3. The characteristic strain as a function of frequency for the postmerger GW signal for irrotational cases with different EOSs (computed in a $\approx 20 \mathrm{~ms}$ window following the peak GW luminosity signal) as seen by an observer oriented face-on (left) or edge-on (right) with respect to the orbital plane. All $2 \leq \ell \leq 6$ modes are used in the plot. The $m=1$, and $m=-1$ modes are those driving the peak at $\mathrm{f} \sim 1.6 \mathrm{kHz}$ in the right panel. The case with the HB EOS collapses to a $\mathrm{BH}$ during this time. 

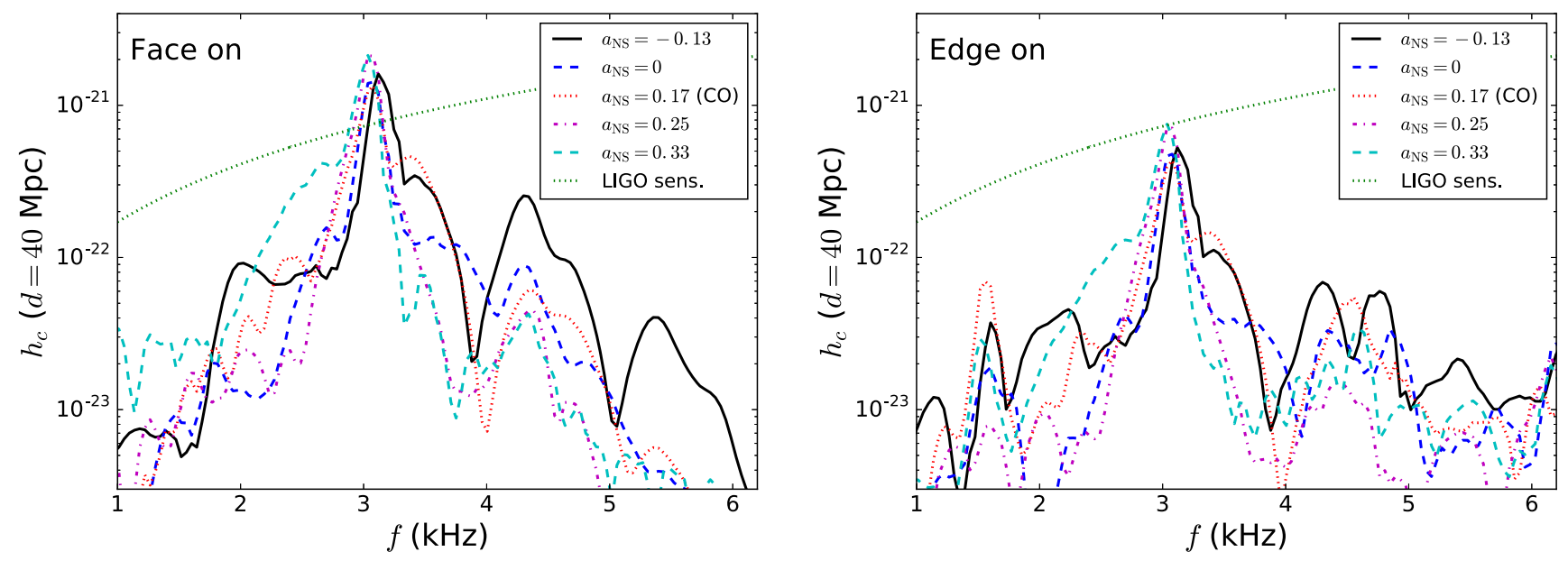

FIG. 4. The characteristic strain as a function of frequency for the postmerger GW signal for cases with the ENG EOS and various spins (computed in a $\approx 20 \mathrm{~ms}$ window following the peak GW luminosity signal) as seen by an observer oriented face-on (left) or edgeon (right) with respect to the orbital plane. All $2 \leq \ell \leq 2$ modes are used in the plot. The $m=1$, and $m=-1$ modes are those driving the peak at $f \sim 1.6 \mathrm{kHz}$ in the right panel.

monochromatic emission can persist for much longer than the $20 \mathrm{~ms}$ windows used for generating Fig. 4, building more power. The largest amplitude among the different cases occurs for spinning cases, and in particular for the corotating configuration with the ENG EOS. We can estimate the approximate strength of the long-lived gravitational signal in this case as follows. If we assume that the source is observed on edge and that the $m=1$ mode has constant frequency and amplitude, the signal-to-noise ratio (SNR) for the $m=1$ mode can be estimated via Eq. (81) of [87] (see also [50]) and approximating the $\ell=2, m=1$ mode GW strain as $h_{21} \sim C_{21} /\left(2 \pi f_{m=1}\right)^{2}$

$$
\begin{aligned}
\mathrm{SNR}_{\mathrm{LIGO}} \approx & 3\left(\frac{7 \times 10^{-24} \mathrm{~Hz}^{-1 / 2}}{\sqrt{S_{n}\left(f_{m=1}\right)}}\right)\left(\frac{C_{21} r M}{10^{-4}}\right)\left(\frac{1.6 \mathrm{kHz}}{f_{m=1}}\right)^{2} \\
& \times\left(\frac{T_{m=1}}{100 \mathrm{~ms}}\right)^{1 / 2}\left(\frac{10 \mathrm{Mpc}}{r}\right)
\end{aligned}
$$

for the LIGO zero-detuned high power configuration at design sensitivity. Here, $S_{n}\left(f_{m=1}\right)$ is the detector noise spectral density at the frequency of the one-arm mode, and we adopt a mode lifetime of $T_{m=1}=100 \mathrm{~ms}$ (order of magnitude consistent with some of our simulations) and distance to the source $r=10 \mathrm{Mpc}$. Such high frequency GW signals will only be seen by LIGO for very close events. However, the prospects for third-generation detectors are better: e.g., the Einstein Telescope ET-D configuration [88] would have $10 \times$ the SNR, and there are proposals for obtaining comparable sensitivities in the kilohertz regime by modifying existing ground-based detectors [52]. The lifetime of the one-arm mode may be considerably extended in the cases of initial prograde NS spin due to the additional centrifugal support by the increased total angular momentum which can extend the lifetime of the remnant. Since the one-arm mode decays very slowly, if the remnant survives collapse to a $\mathrm{BH}$ for longer times, the mode may survive for longer times. Note that, as pointed out in [50], detection of the inspiral GW from a BNS will substantially lower the SNR requirements to claim a detection of a post-merger $l=2, m=1 \mathrm{GW}$ mode. In addition, coherent stacking data analysis methods can be adopted to boost the signal from the detection of a number of sources [89] (see also [90]).

\section{B. Properties of the merger remnants}

For the NS merger with the HB EOS, the post-merger remnant collapses to a $\mathrm{BH}$ with $M_{\mathrm{BH}} \approx 0.94 M$ and a dimensionless spin of $a_{\mathrm{BH}} \approx 0.6$ during the simulation. For all other cases, the remnant star lasts the entire extent of our simulations. In the following we discuss the angular velocity profiles and nonaxisymmetric density modes that are excited in these remnants.

\section{Angular velocity}

The remnant stars in our simulations settle into a slowly varying pattern of differential rotation. In recent studies, it has been found that the angular velocity profile $(\Omega$ versus the cylindrical radius $\varpi$ ) in massive BNS merger remnants formed from initially irrotational configurations appears to have an approximately universal shape independent of initial data, gauge conditions, equations of state, and initial spin (when starting with constraint-violating and nonequilibrium initial data). This angular velocity profile is constant near the center of the star, then as $\varpi$ increases, $\Omega$ increases until it reaches a maximum value, after which $\Omega$ falls off with $\varpi[44,45,85,91-96]$. Here we test whether this profile depends on the initial NS spin, when starting with constraint-satisfying and equilibrium initial data. 


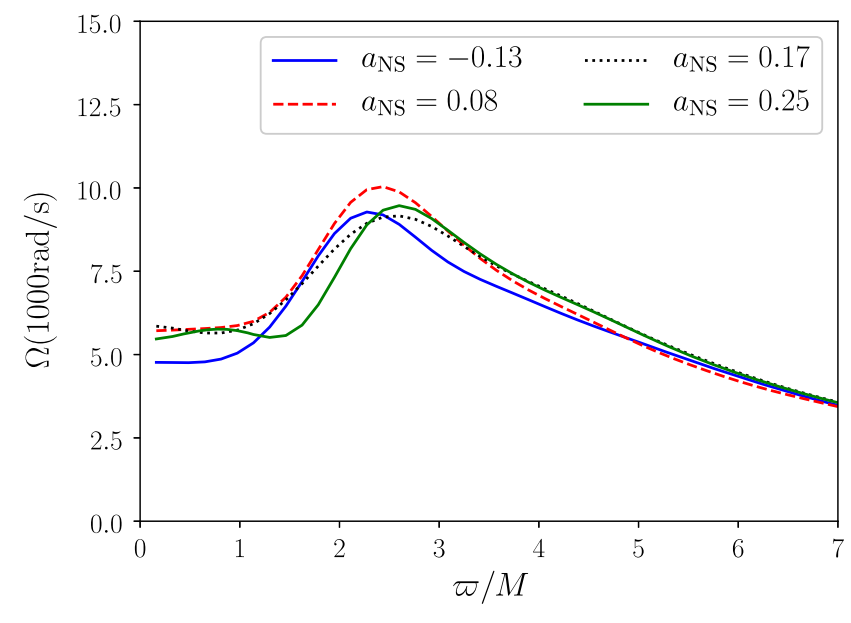

FIG. 5. The angular velocity versus cylindrical coordinate radius on the equator of the massive NS remnant for select cases with the ENG EOS. The $a_{\mathrm{NS}}=0.17$ case shown corresponds to the corotating initial data.

In Fig. 5, we show the late-time azimuthally averaged angular velocity profiles of cases with different initial spins. The late-time rotational profiles appear to be relatively insensitive to the initial spin. However, we point out that these calculations are not gauge invariant, nor is the initial center of mass the true center of rotation of these configurations. Moreover, within several tens of ms from merger, magnetic fields can brake the differential rotation, bringing the core to a near uniformly rotating state [40]. Thus, these $\Omega$ profiles are not expected to be long lived, even if the remnant survives for long times.

\section{Nonaxisymmetric instabilities}

We also quantify how the azimuthal matter distribution of the post-merger remnant evolves with time. In Fig. 6, we

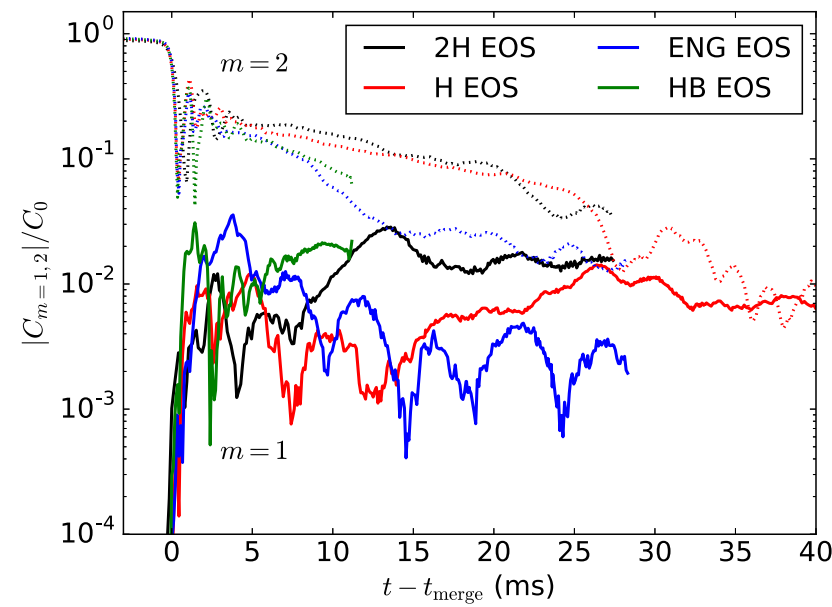

show the magnitude of the $m=1$ and 2 azimuthal density modes. Since all cases considered here initially have identical binary constituents, the $m=1$ component is essentially zero pre-merger, but is generated by shear instabilities during the merger. All cases excite the $m=$ 1 mode, but for several cases-namely the irrotational $2 \mathrm{H}$ and H EOSs, as well as the ENG $a_{\mathrm{NS}}=-0.13, a_{\mathrm{NS}}=0.17$ CO (and SP to a slightly lesser extent; see Appendix A), and $a_{\mathrm{NS}}=0.33$ - the combination of the persistence of the merger-generated $m=1$ mode combined with the more rapid decay of the $m=2$ density perturbation means that the former is comparable or greater in magnitude to the latter at late times. For the ENG $a_{\mathrm{NS}}=-0.13$, IR $\left(a_{\mathrm{NS}}=0\right), \mathrm{CO} a_{\mathrm{NS}}=0.17$, and $a_{\mathrm{NS}}=0.33$ cases, we have also Fourier transformed the $m=1$ density modes shown in Fig. 6, and found that the peak $m=1$ frequencies occur at $1656 \mathrm{~Hz}, 1655 \mathrm{~Hz}, 1593 \mathrm{~Hz}$, and $1584 \mathrm{~Hz}$, respectively. These frequencies agree well with the frequencies of the corresponding peaks at $\sim 1600 \mathrm{~Hz}$ in the GW spectrum shown on the right panel in Fig. 4, indicating that it is the $m=1$ mode that is driving that peak. In addition, these frequencies demonstrate that as the pre-merger NS spin increases the additional angular momentum shifts the onearm mode frequency to smaller values.

Therefore, our study suggests that the $m=1$ instability, as found in eccentric mergers [47-49] and certain irrotational quasicircular binary mergers including unequal mass cases [50,51], can also arise in equal mass quasicircular BNS mergers including spin, and leaves a similar imprint on the GW spectrum. However, we do not find a particular trend of the mode amplitude with initial spin.

We note that the growth of the $m=1$ instability can be tracked in a gauge invariant way through the mode decomposition of the GWs. In cases we study here, the amplitude of $m=1 \mathrm{GW}$ modes grows rapidly during merger and saturates post-merger, as we have found in

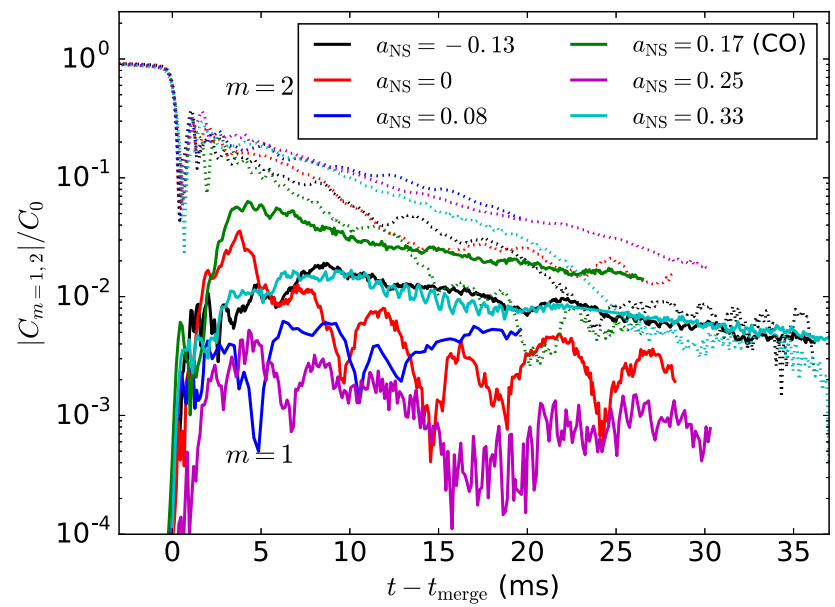

FIG. 6. The magnitude of the $m=1$ and $m=2$ azimuthal density modes as a function of time for nonspinning cases with various EOSs (left) and for the ENG EOS with various values of NS spin (right). The curves for the HB EOS end at the time of BH formation; the curves for the other cases end when their respective simulations were stopped. 

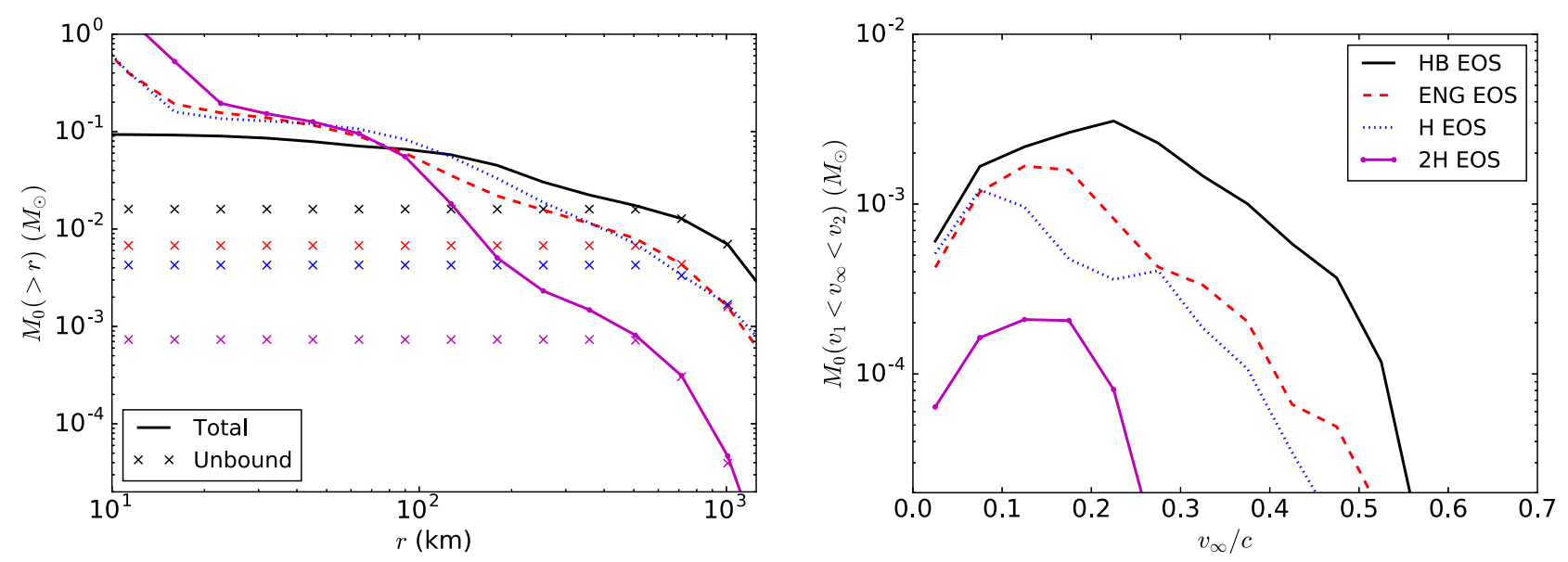

FIG. 7. Left: The amount of rest mass outside a given coordinate radius from the center of mass for the post-merger remnant for mergers of nonspinning NSs with various EOSs. The solid curves show the total amount, while the points show just the unbound rest mass. Right: The amount of unbound rest mass binned by the velocity at infinity, with each bin $0.05 c$ in size. The legend from the right panel applies to the left panel as well.

previous studies (see Fig. 13 in Ref. [48] and Fig. 2 in Ref. [49]).

\section{Post-merger matter distribution and electromagnetic counterparts}

In this section, we examine the post-merger distribution of matter. Comparing nonspinning cases with different EOSs, we see in Fig. 7 that stiffer EOSs give rise to more spread out distributions of bound matter, but produce less unbound matter compared to softer EOSs. This trend in ejecta has been noted in numerous studies of BNS mergers (see, e.g., [97-99], and [100] for a review), and can be attributed to the fact that smaller radius NSs collide at higher velocities, and thus produce more shock-heated dynamical ejecta.

The trend in unbound matter with NS spin is less clear cut. As shown in Fig. 8, the greatest amount of unbound material for the ENG EOS $\left(\sim 2 \% M_{\odot}\right)$ is found for spins antialigned with the orbital angular momentum. A similar result was also reported in [41]. Our cases with increasing spin aligned with the orbital angular momentum show decreasing amounts of unbound material up to $a_{\mathrm{NS}} \approx 0.17$, at which point the trend reverses, with the highest spin cases showing increased amounts of unbound material up to $a_{\mathrm{NS}}=0.33$, which shows a similar amount of unbound material to the nonspinning case. The fact that higher negative spin results in more ejected matter is likely related with the fact that in such cases the NSs plunge and collide at larger velocities, ejecting more matter. On the other hand, for sufficiently rapidly rotating NSs (certainly as one approaches breakup spins) one can also anticipate that it will be easier to tidally unbind the outer NS matter.

In addition to affecting the total amount of mass of the ejecta, and the ejecta mass distribution with velocity, spin substantially impacts the angular distribution of dynamical
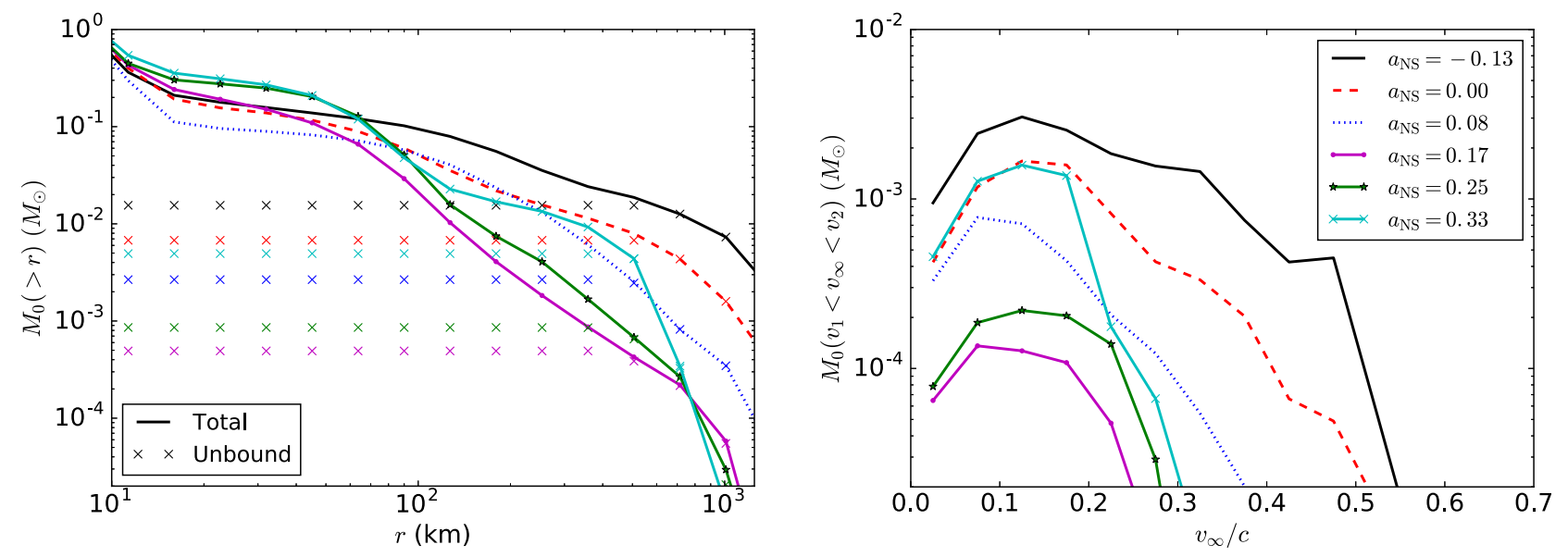

FIG. 8. Same as Fig. 7, but for cases with the ENG EOS and various values of NS spin. The legend from the right panel applies to the left panel as well. 

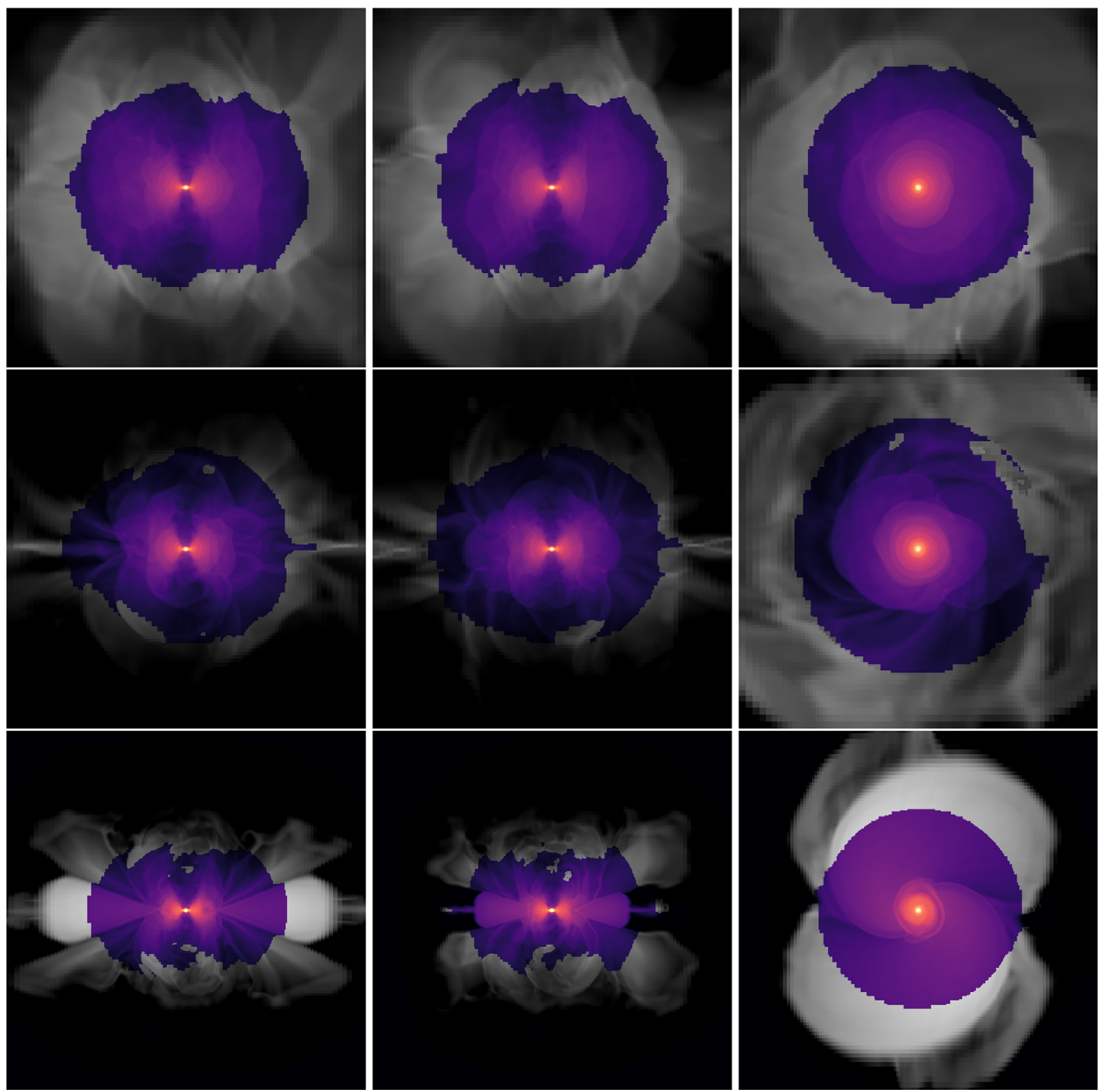

FIG. 9. The rest-mass density distribution $\left(D:=\rho_{0} u^{t} \sqrt{-g}\right)$ of the bound (inner region; purple-yellow color scale) and unbound matter (outer region; black-white color scale) $\sim 10 \mathrm{~ms}$ after merger. The columns show (left to right) slices in the $x=0, y=0$, and $z=0$ (equatorial) plane. The rows (top to bottom) show cases with $a_{\mathrm{NS}}=0,0.17(\mathrm{CO})$, and 0.33 . The plots show roughly $1800 \mathrm{~km}$ in each linear dimension.

ejecta. In Fig. 9, we show snapshots of rest-mass density on the $x=0$ (left column), $y=0$ (middle column), and $z=0$ (equatorial; right column) planes with the zero pre-merger spin on the top row, $a_{\mathrm{NS}}=0.17$ in the middle row, and $a_{\mathrm{NS}}=0.33$ on the bottom row. The figure demonstrates that as the aligned spin increases, the unbound matter becomes more concentrated near the orbital plane, consistent with being due to tidal effects. By contrast, for smaller spins, the ejecta are more isotropically distributed.

A similar result showing that antialigned spin increased the amount of dynamical ejecta, while aligned spins up to $\sim 0.3$ decreased the amount ejecta and caused it to be more concentrated toward the equatorial plane, was also found recently in Ref. [41]. In contrast to what we find here, in that case no enhancement in ejecta was found for very high spins. However, [41] used finite-temperature EOSs (and slightly lower maximum spin values), and so is not directly comparable to this work. Moreover, Ref. [45] did not find the greatest amount of ejecta for the antialigned spin case. However, that work not only used different EOSs, but also constraint-violating and nonequilibrium initial data, so that work is also not directly comparable to the present study.

Apart from larger dynamical ejecta masses, we also find that NS spins that are antialigned with the orbital angular momentum lead to a substantial amount of matter moving outward at higher velocities (see right panel of Fig. 8). 
By contrast, larger aligned spin decreases the width of the velocity distribution up to the spin value of 0.17 , above which the distribution width increases again. The fact that in the antialigned case there is a tail of the ejecta moving at high velocities implies that the radio emission from antialigned spin BNS mergers can be significantly brighter compared to irrotational mergers. In particular, Fig. 8 suggests that in the antialigned case about $10^{-3} M_{\odot}$ of matter moves at speed greater than $0.3 \mathrm{c}$, which implies that this tail alone has a total kinetic energy of about $10^{51} \mathrm{erg}$. Therefore, based on Eqs. (4) and (5), mergers with antialigned spin are likely to have radio emission from the interaction with the interstellar medium which is significantly brighter with shorter rise times than irrotational and aligned-spin cases.

This trend of the ejecta mass distribution and ejecta velocities with spin would cause the anticipated kilonova component that is powered by dynamical ejecta to be significantly brighter in the antialigned case. In addition, the estimated kilonova in the irrotational case is brighter than all cases with aligned spin that we study. The latter results holds despite the fact that the amount of dynamically ejected mass in the spin 0.33 and irrotational cases is approximately the same. We detail the properties of the unbound material in Table II, along with an estimate of how these may translate into differences in the ejecta-powered kilonovae by use of Eq. (3). As can be seen in the table, keeping all the other parameters fixed, NS spin can make an order of magnitude difference in the ejecta properties, including the kilonova rise time. However, its influence is degenerate with other parameters, such as the NS EOS, and most likely also depends on the mass ratio, which we do not treat here. We also note that there is almost a factor of 2 difference in the amount of unbound material for the two different $a_{\mathrm{NS}}=0.17$ cases, though in both cases the total mass is small compared to the other cases.
In recent years, a number of studies [78-84] have suggested that a large fraction of the mass of the disk that forms around the BNS merger remnant becomes unbound due to the effects of viscosity, neutrinos, and/or magnetic fields. These disk winds contribute to the kilonova signature, and appear necessary to explain GW170817. Therefore, the amount of mass that forms a disk around the merger remnant crucially determines the kilonova properties, as well as, presumably, affecting any accompanying gamma-ray burst. In Table II, we list the amount of matter in the disk around the merger remnant. As with the dynamical ejecta, the trend of the disk mass with spin is not monotonic. However, the highest aligned-spin case has the most massive accretion disk. This result is in agreement with the findings of [40] where a simpler $\Gamma$-law EOS with $\Gamma=2$ was adopted, but magnetic fields were treated. Here we find that, compared to the irrotational case, dimensionless spins of $\sim 0.3$ can double the amount of mass in the disk around the merger remnant. Therefore, high aligned spin makes it easier to form large accretion disks that can contribute to the blue component of kilonovae.

\section{CONCLUSIONS}

In this work, we presented results from fully relativistic hydrodynamic simulations of quasiequilibrium BNSs in quasicircular orbits with spinning components. We considered configurations of equal mass and equal spin, with initial dimensionless NS spins in the range $a_{\mathrm{NS}} \in[-0.13,0.33]$. We modeled the matter as a perfect fluid, with different piecewise polytropic representations for the equation of state, covering a range of compactness for a $1.4 M_{\odot}$ NS from $\sim 0.136$ to 0.178 . We focused on quantifying the effects of pre-merger NS spin, and neglected the effect of magnetic fields, neutrinos, and realistic nuclear microphysics in this first study.

TABLE II. The properties of the bound and unbound NS matter from various cases. The columns list the EOS, the dimensionless NS spins $a_{\mathrm{NS}}$ (which are the same for both stars), the bound rest mass $M_{0 \text {,disk }}$ with $r>32 \mathrm{~km}$ in units of $M_{\odot} / 100$ and the radius $R_{\text {disk }}$ inside which $90 \%$ of this mass is contained in units of $10^{5} \mathrm{~m}$, the unbound rest mass $M_{0}$ in units of $M_{\odot} / 100$, the average asymptotic velocity weighted by rest mass $\left\langle v_{\infty}\right\rangle$, the total kinetic energy in units of $10^{50} \mathrm{erg}$, the anticipated kilonovae rise time in days, and the associated luminosity in units of $10^{41} \mathrm{erg} / \mathrm{s}$, which we compute via Eqs. (2) and (3). See Appendix B for details regarding measuring these quantities.

\begin{tabular}{lcccccccc}
\hline \hline EOS & $a_{\mathrm{NS}}$ & Spin state & $M_{0, \text { disk }}$ & $R_{\text {disk }}$ & $M_{0, \mathrm{u}}$ & $\left\langle v_{\infty}\right\rangle$ & $E_{\text {kin, } 50}$ & $t_{\text {peak }}$ \\
\hline ENG & -0.13 & SP & 14 & 3.1 & 1.55 & 0.20 & 7.88 & 0.37 \\
ENG & 0.00 & IR & 13 & 2.0 & 0.68 & 0.17 & 2.36 & 0.23 \\
ENG & 0.08 & SP & 9 & 2.8 & 0.27 & 0.13 & 0.55 & 0.12 \\
ENG & 0.17 & SP & 15 & 1.2 & 0.05 & 0.12 & 0.08 & 0.05 \\
ENG & 0.17 & CO & 17 & 1.3 & 0.09 & 0.12 & 0.15 & 0.07 \\
ENG & 0.25 & SP & 25 & 1.2 & 0.09 & 0.14 & 0.19 & 0.07 \\
ENG & 0.33 & SP & 26 & 1.1 & 0.49 & 0.12 & 0.81 & 0.17 \\
2H & 0.00 & IR & 15 & 1.4 & 0.07 & 0.13 & 0.14 & 0.07 \\
H & 0.00 & IR & 12 & 2.8 & 0.43 & 0.15 & 1.23 & 0.17 \\
HB & 0.00 & IR & 7 & 3.5 & 1.60 & 0.22 & 9.53 & 0.37 \\
\hline \hline
\end{tabular}


We find that the basic features of the GW spectrum following merger are broadly consistent with what has been found in previous works (see [101] for a review). More specifically, we find that the post-merger peak GW frequency is only weakly influenced by the NS spin (by about 100-200 Hz) consistent with Refs. [35,37,46]. We find that aligned (antialigned) spin cases give slightly lower (higher) values of the post-merger peak GW frequency when compared to the irrotational case. At higher frequencies (next to the peak GW frequency) antialigned configurations enhance the GW power with respect to aligned ones. In turn, these results imply that there is some degree of degeneracy between pre-merger spin and the nuclear EOS, and without some independent measurement of spin this should contribute to the systematic error when inferring the EOS from the post-merger peak GW frequency.

We find that the one-arm instability can also operate in the remnant of quasicircular mergers with spin. Spin affects the GW frequency associated with the one-arm mode in the same way it affects the peak post-merger GW frequency, i.e., aligned spin shifts the one-arm mode frequency to lower values. Our studies do not show a significant correlation between the energy that goes into the onearm mode and the pre-merger spin. We find that the strongest one-arm mode develops for an initially corotating case that we consider. The GW signal from this one-arm mode could be detectable by third-generation GW detectors, and the one-arm mode GW frequency can be used to infer properties of the nuclear EOS.

Our simulations demonstrate that spin has a substantial impact on the mass, velocity, and angular distribution of dynamical ejecta, that would likely be reflected in the red kilonova signatures from such events. Our results also indicate that spins antialigned with the orbital angular momentum result in more massive dynamical ejecta, with a considerable amount of matter traveling at speeds near $0.5 \mathrm{c}$ (see also [41]). Antialigned spin mergers generate brighter red kilonovae than aligned-spin cases, because the latter have suppressed dynamical ejecta masses. However, we find that as the aligned spin increases past a certain value, the amount of dynamical ejecta increases again (but the velocity distribution of ejecta masses is narrower than the irrotational and antialigned cases). This implies that the expected red kilonovae should again become brighter as the spin increases-consistent with the fact that as the spin frequency increases, the star becomes less bound, and hence it becomes easier to dynamically eject more mass. For higher spins, we find that the dynamical ejecta are more concentrated around the orbital plane.

The blue kilonova expected from unbinding part of the remnant disk is also affected by the initial NS spin insofar as the latter affects the disk mass and/or the lifetime of the remnant. Recent work $[84,102,103]$ has shown that a substantial amount of mass outside the remnant becomes unbound due to viscous/magnetic/neutrino processes.
For aligned dimensionless spins of $\sim 0.2-0.3$, the merger remnants have larger disks than the lower-spin cases, and the massive NS remnants from aligned-spin BNS mergers likely survive for longer times than those from nonspinning mergers due to the extra centrifugal support provided by the additional total angular momentum. The longer the massive NS remnant survives, the larger the unbound disk mass due to strong neutrino irradiation from the hot remnant can be. Also, assuming that the fraction of the disk mass that becomes unbound is approximately independent of the mass of the disk that initially forms around the remnant, we anticipate that the blue kilonovae should be brighter in NS mergers with higher aligned initial spins. However, this conjecture should be carefully studied with long-term viscous/MHD studies that treat neutrino heating. Studies which attempt to place a constraint on the binary tidal deformability $\tilde{\Lambda}$ based on how much mass goes into a disk structure have so far not included the effects of spin. For example, the work of [16] suggests that for equal mass binaries, $\tilde{\Lambda} \gtrsim 400$ is necessary to explain the kilonova counterpart to GW170817, while this value is lowered in the unequal mass case [53]. These studies may need to be revisited to include the effects of initial NS spin, and the bounds on $\tilde{\Lambda}$ are likely to become less restrictive for spinning BNSs. We point out that in our work we do not study asymmetric binaries, e.g., unequal masses or unequal spins, nor do we treat magnetic fields, neutrinos, or other such microphysics effects, all of which could change some of our results. We intend to address these points in future work.

Finally, we compared two simulations that have the same initial properties, i.e., the same total mass, orbital angular frequency, and circulation, but one corresponds to corotating initial data and the other to the corotating counterpart constructed with the constant rotational-velocity formulation $[55,56]$. The two binary systems are broadly equivalent, though we find some differences in the merger time and post-merger matter distribution. This could be due to the fact that their identification through the concept of equatorial circulation is not exact [57]. In turn the outer layers of the stars may have slightly different properties, which would lead to observed differences in dynamical ejecta masses, etc.

\section{ACKNOWLEDGMENTS}

W.E. E. acknowledges support from an NSERC Discovery grant. V.P. acknowledges support from NSF Grant No. PHY-1912619. F. P. acknowledges support from NSF Grant No. PHY-1607449, the Simons Foundation, and the Canadian Institute For Advanced Research (CIFAR). A. T. acknowledges support from NSF Grant No. PHY1662211, and NASA Grant No. 80NSSC17K0070. V.P. and F. P. would like to thank KITP for hospitality, where part of this work was completed. V. P. would also like to thank B. Metzger for useful discussions. This research was supported in part by Perimeter Institute for Theoretical Physics. 
Research at Perimeter Institute is supported by the Government of Canada through the Department of Innovation, Science and Economic Development Canada and by the Province of Ontario through the Ministry of Economic Development, Job Creation and Trade. Research at K. I. T.P. is supported in part by the National Science Foundation under Grant No. NSF PHY-1748958. Computational resources were provided by Extreme Science and Engineering Discovery Environment (XSEDE) under Grant No. TG-PHY100053 and the Perseus cluster at Princeton University.

\section{APPENDIX A: COROTATING VERSUS SPINNING INITIAL DATA}

Here we compare the two cases we consider that have the same quasilocal value for the dimensionless NS spin,
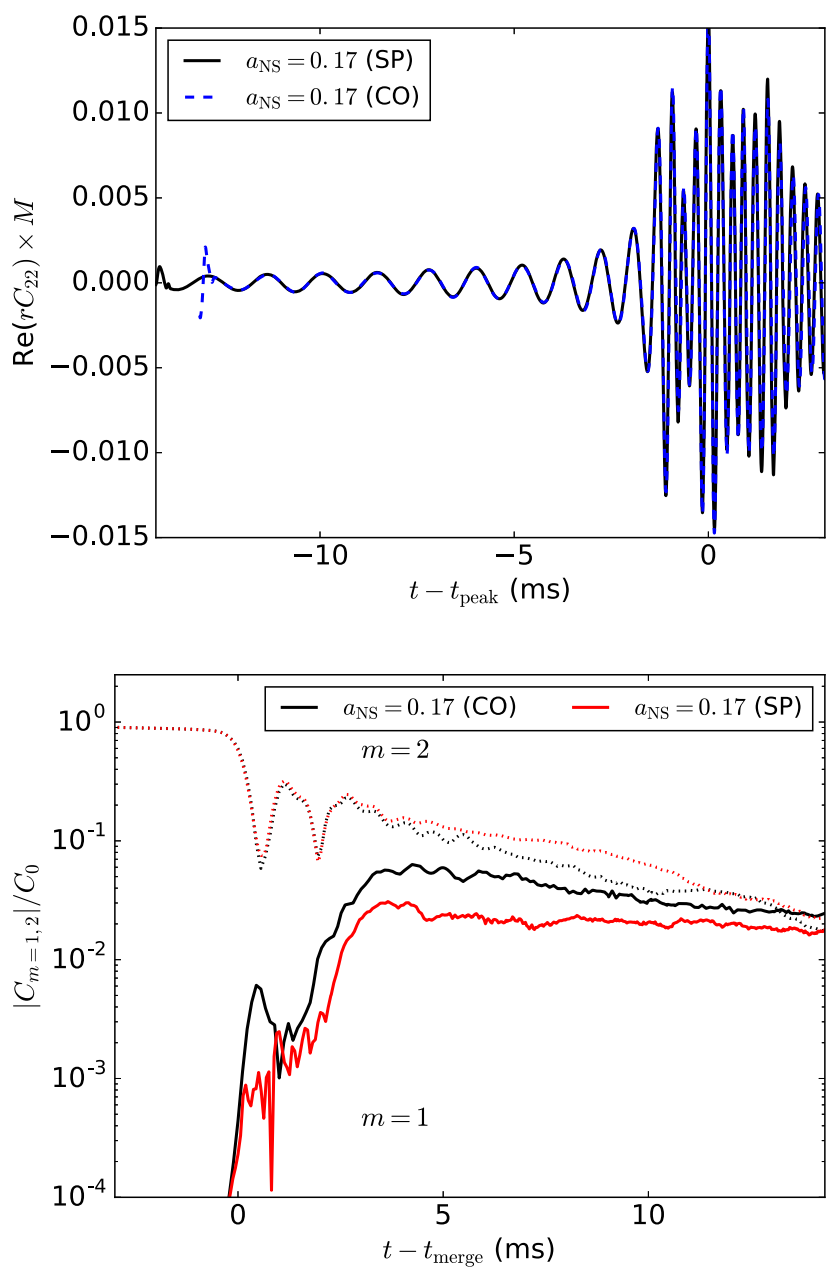

FIG. 10. Top: The GW signal $\left(\ell=m=2\right.$ component of $\psi_{4}$ multiplied by the extraction radius $r$ ) from mergers with the ENG EOS and $a_{\mathrm{NS}}=0.17$ but different spin formalisms. The curves have been aligned in time and phase at peak. In particular, the $\mathrm{CO}$ case has been shifted ahead by $1.1 \mathrm{~ms}$ relative to the SP case. Bottom: The magnitude of the $m=1$ and $m=2$ azimuthal density modes as a function of time for the same two cases. namely, the ENG $a_{\mathrm{NS}}=0.17$ "SP" and "CO" cases listed in Table I. These two types of initial configurations correspond to different formalisms, but their global properties are essentially the same.

Despite the global similarities in the two types of initial data, we find that their evolution has some differences. To begin with, the SP case undergoes an additional $\sim 1 / 3$ of orbit before merging, though accounting for this difference in the time (and phase) of the merger, the GW signals otherwise line up well as shown in the top panel of Fig. 10. The disk mass that forms in the two cases differs by about $10 \%$, but the total dynamical ejecta in the $\mathrm{CO}$ case is about 2 times as massive as in the SP case (though in either case it is small). The average ejecta velocity is the same in the two cases, but the kinetic energy is a factor of 2 different. There is also some difference between the amplitudes of the azimuthal density modes produced post-merger, with the SP case exhibiting slightly larger $m=2$ and slightly smaller $m=1$ modes relative to the $\mathrm{CO}$ case, as shown in bottom panel of Fig. 10. However, higher-resolution simulations are probably required to determine how much of this difference is numerical.

The small differences between the two types of initial data suggest that the "corotation limit" of the constant rotationalvelocity formulation agrees well with the corresponding corotating formulation. The bulk of the matter behaves the same in the two formulations, but it is likely that the outer NS layers in the constant rotational-velocity formulation have slightly different properties, probably as a result of their identification through their circulation. In turn this would explain the difference in dynamical ejecta that we find.

\section{APPENDIX B: NUMERICAL ERRORS AND CONVERGENCE STUDY}

As noted in the main text, for select cases we perform a resolution study utilizing $4 / 3 \times$ and $2 \times$ the default resolution, which has $d x \approx 0.05 M$ on the finest level of mesh refinement. In Figs. 11 and 12, we show, respectively, how the amplitude and phase of the GWs vary with resolution for the merger of nonspinning NSs and the merger with the highest value of NS spin $\left(a_{\mathrm{NS}}=0.33\right)$ for the ENG EOS. In both cases, we can see that leading up to merger the differences across resolutions are small, though postmerger the phase errors become significant, as is typically found in BNS simulations. Examining the resolution dependence of the peak frequency of the post-merger GWs for these two cases, we find the differences between the two lower resolutions (the ones we continue for at least $10 \mathrm{~ms}$ post-merger) to be small (on the order of $\mathrm{Hz}$ ) compared to the differences due to NS spin.

Notice that Fig. 11 explicitly demonstrates that the postmerger GW amplitude for the highest spinning case has a small dependence on numerical resolution for the parameters used here. By contrast, the irrotational case has a more significant resolution dependence post-merger. However, 

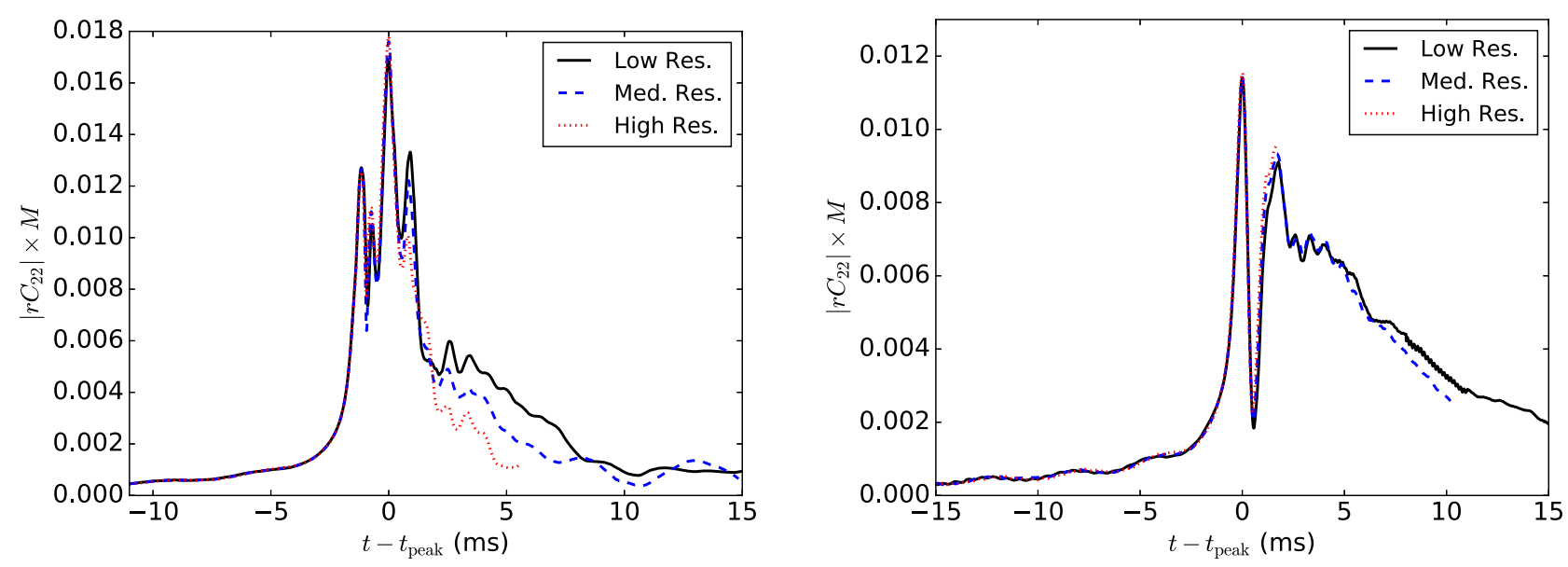

FIG. 11. The GW amplitude (magnitude of $\ell=m=2$ component of $\psi_{4}$ multiplied by the extraction radius $r$ ) from mergers with the ENG EOS, with nonspinning NSs shown on the left, and the largest value of NS spin considered $\left(a_{\mathrm{NS}}=0.33\right)$ shown on the right. Three different resolutions are plotted, and the curves have been aligned in time at peak amplitude. The phase error is shown in Fig. 12.
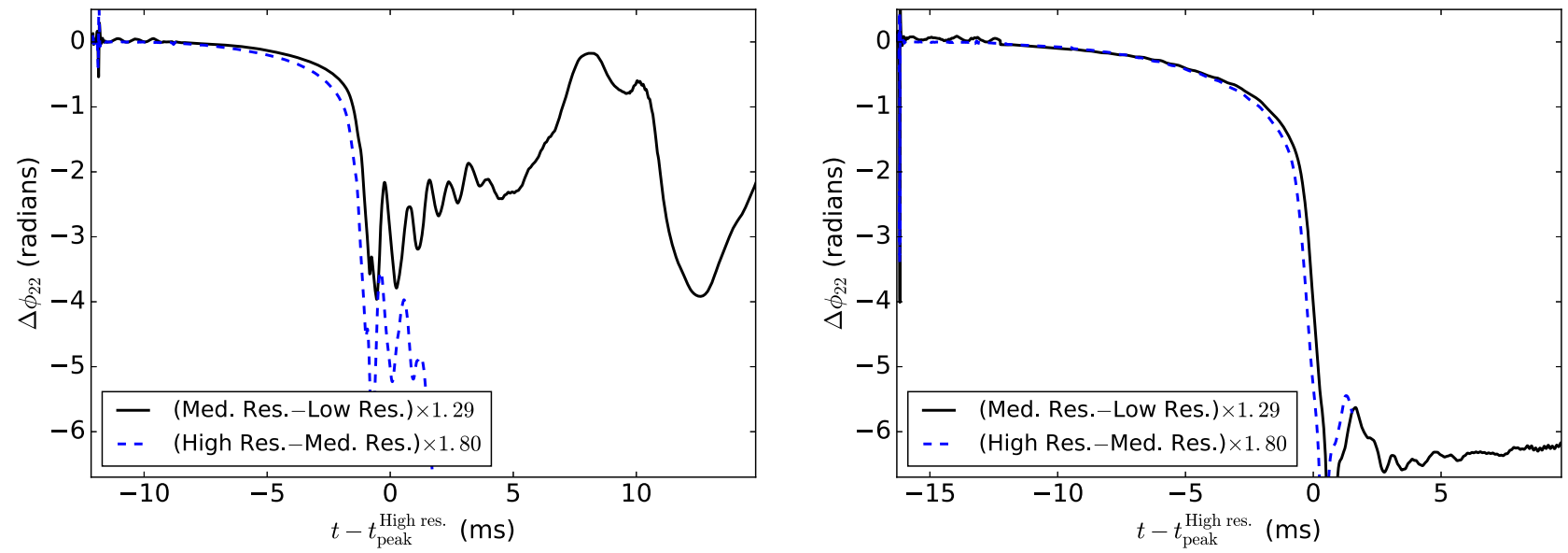

FIG. 12. The difference in the GW phase (phase of $\ell=m=2$ component of $\psi_{4}$ ) across different resolutions from mergers with the ENG EOS and nonspinning NSs (left), and the largest value of NS spin considered $\left(a_{\mathrm{NS}}=0.33\right.$; right). The differences have been scaled assuming second order convergence. Time is shown on the vertical axis with respect to where the peak of the GW signal occurs in the highest resolution case (in particular, the GWs have not been aligned at merger).

the nonspinning cases show that the higher the resolution, the faster the post-merger GW amplitude decays. Therefore, we can conclude that the post-merger GW amplitude in nonzero spin cases decays more slowly in the first $20 \mathrm{~ms}$ than in the irrotational case, as stated in the main text.

We also show the convergence of the constraint violation, leading up to and post-merger in Fig. 13. The convergence towards zero with resolution of this quantity is approximately consistent with second order, as expected.

Finally, we comment on the measurement of the bound/ unbound matter. In Fig. 14 we show how this depends on time for an example case. From this it can be seen that these quantities are relatively constant for $t-t_{\text {merge }}>10 \mathrm{~ms}$ (e.g., the total unbound matter increases by $<5 \%$ ), with the material marked as unbound moving outward in radius as expected, while maintaining the same distribution in velocity (at infinity). The results shown in the main text are from the latest time shown in Fig. 14.

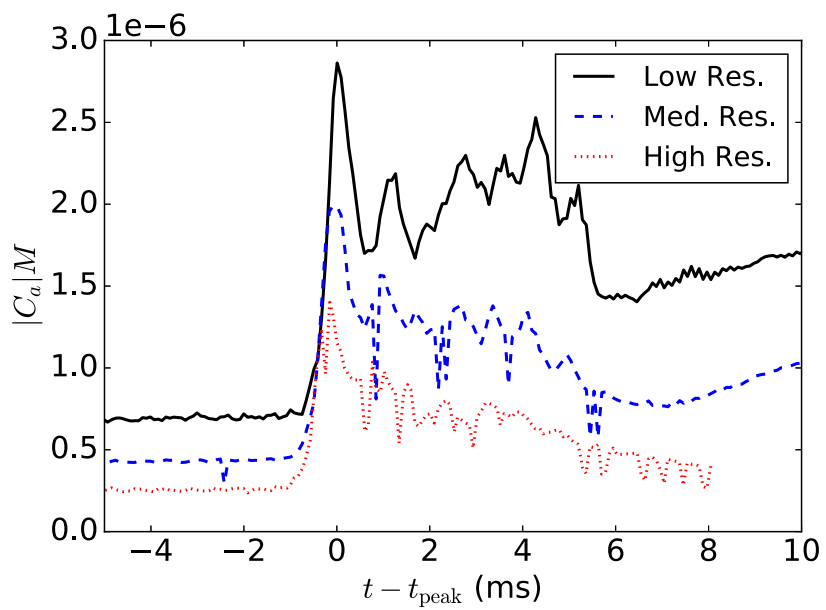

FIG. 13. The norm of the generalized-harmonic constraint $C^{a}=\square x^{a}-H^{a}$ integrated in the equatorial plane for several different resolutions for the IR, ENG EOS case. 

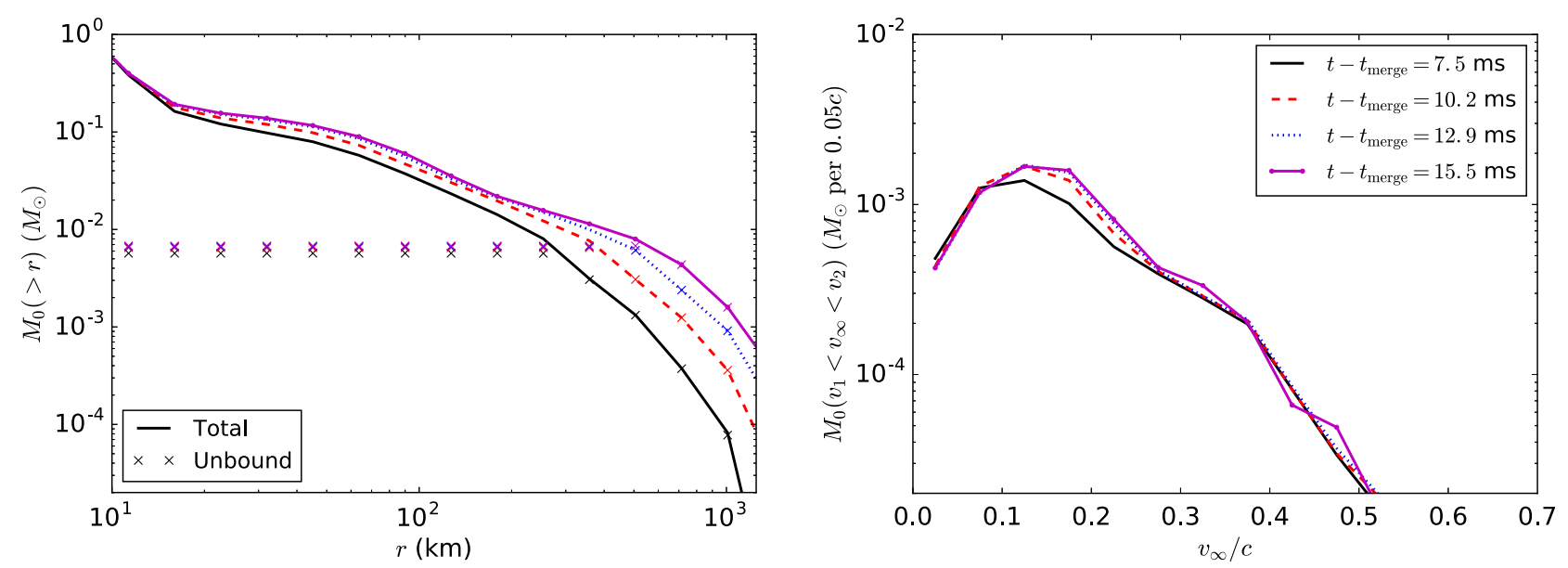

FIG. 14. Same as Fig. 8, but just showing the case with the ENG EOS and no NS spin at different times post-merger. The legend from the right panel applies to the left panel as well.

We have not run the highest resolution case sufficiently long to compute this diagnostic, but comparing the lower two resolutions, we find a relative difference of $\approx 9 \%$ in the unbound material for the ENG EOS nonspinning case, and a somewhat larger difference of $30 \%$ in the $a_{\mathrm{NS}}=0.33$ case. For reference we note that we use a so-called "artificial atmosphere," as is typical in such hydrodynamical simulations, that has a maximum density of $\approx 8 \times$ $10^{4} \mathrm{gm} / \mathrm{cm}^{3}$ (i.e., roughly 10 orders of magnitude below the maximum density of the NSs) and gradually decreases in density towards the outer boundary, as described in Ref. [58].
[1] B. P. Abbott et al. (LIGO Scientific and Virgo Collaboration), Phys. Rev. Lett. 116, 061102 (2016).

[2] B. P. Abbott et al. (Virgo and LIGO Scientific Collaborations), Phys. Rev. Lett. 116, 241103 (2016).

[3] B. P. Abbott et al. (Virgo and LIGO Scientific Collaborations), Phys. Rev. Lett. 118, 221101 (2017).

[4] B. P. Abbott et al. (Virgo and LIGO Scientific Collaborations), Phys. Rev. Lett. 119, 141101 (2017).

[5] B. P. Abbott et al. (Virgo and LIGO Scientific Collaborations), Astrophys. J. 851, L35 (2017).

[6] B. P. Abbott et al. (LIGO Scientific and Virgo Collaborations), arXiv:1811.12940.

[7] B. P. Abbott et al. (Virgo and LIGO Scientific Collaborations), Phys. Rev. Lett. 119, 161101 (2017).

[8] B. P. Abbott et al. (SKA South Africa/MeerKAT Collaboration), Astrophys. J. 848, L12 (2017).

[9] B. P. Abbott et al. (Virgo, Fermi-GBM, INTEGRAL, and LIGO Scientific Collaborations), Astrophys. J. 848, L13 (2017).

[10] B. Margalit and B. D. Metzger, Astrophys. J. Lett. 850, L19 (2017).

[11] M. Shibata, S. Fujibayashi, K. Hotokezaka, K. Kiuchi, K. Kyutoku, Y. Sekiguchi, and M. Tanaka, Phys. Rev. D 96, 123012 (2017).

[12] V. Paschalidis, K. Yagi, D. Alvarez-Castillo, D. B. Blaschke, and A. Sedrakian, Phys. Rev. D 97, 084038 (2018).
[13] A. Bauswein, O. Just, H.-T. Janka, and N. Stergioulas, Astrophys. J. 850, L34 (2017).

[14] M. Ruiz, S. L. Shapiro, and A. Tsokaros, Phys. Rev. D 97, 021501 (2018).

[15] E. Annala, T. Gorda, A. Kurkela, and A. Vuorinen, Phys. Rev. Lett. 120, 172703 (2018).

[16] D. Radice, A. Perego, F. Zappa, and S. Bernuzzi, Astrophys. J. 852, L29 (2018).

[17] E. R. Most, L. R. Weih, L. Rezzolla, and J. SchaffnerBielich, Phys. Rev. Lett. 120, 261103 (2018).

[18] C. Raithel, F. zel, and D. Psaltis, Astrophys. J. 857, L23 (2018).

[19] B. P. Abbott et al. (Virgo and LIGO Scientific Collaborations), Phys. Rev. Lett. 121, 161101 (2018).

[20] E. Annala, T. Gorda, A. Kurkela, J. Nttil, and A. Vuorinen, arXiv:1903.09121.

[21] G. Bozzola, P. L. Espino, C. D. Lewin, and V. Paschalidis, Eur. Phys. J. A 55, 149 (2019).

[22] S. Gandolfi, J. Lippuner, A. W. Steiner, I. Tews, X. Du, and M. Al-Mamun, J. Phys. G 46, 103001 (2019).

[23] C. A. Raithel, Eur. Phys. J. A 55, 80 (2019).

[24] E. Pian et al., Nature (London) 551, 67 (2017).

[25] B. Abbott et al. (LIGO Scientific, Virgo, Las Cumbres Observatory, 1M2H, DLT40, MASTER, VINROUGE, Dark Energy Camera GW-EM, and DES Collaborations), Nature (London) 551, 85 (2017). 
[26] L. Lombriser and A. Taylor, J. Cosmol. Astropart. Phys. 03 (2016) 031.

[27] L. Lombriser and N. A. Lima, Phys. Lett. B 765, 382 (2017).

[28] T. Baker, E. Bellini, P. G. Ferreira, M. Lagos, J. Noller, and I. Sawicki, Phys. Rev. Lett. 119, 251301 (2017).

[29] L. Heisenberg and S. Tsujikawa, J. Cosmol. Astropart. Phys. 01 (2018) 044.

[30] P. Creminelli and F. Vernizzi, Phys. Rev. Lett. 119, 251302 (2017).

[31] J. M. Ezquiaga and M. Zumalacrregui, Phys. Rev. Lett. 119, 251304 (2017).

[32] J. Sakstein and B. Jain, Phys. Rev. Lett. 119, 251303 (2017).

[33] W. E. East, V. Paschalidis, and F. Pretorius, Astrophys. J. Lett. 807, L3 (2015).

[34] V. Paschalidis, Classical Quantum Gravity 34, 084002 (2017).

[35] S. Bernuzzi, T. Dietrich, W. Tichy, and B. Bruegmann, Phys. Rev. D 89, 104021 (2014).

[36] N. Tacik et al., Phys. Rev. D 92, 124012 (2015); 94, 049903(E) (2016).

[37] T. Dietrich, S. Bernuzzi, M. Ujevic, and W. Tichy, Phys. Rev. D 95, 044045 (2017).

[38] T. Dietrich, N. Moldenhauer, N. K. Johnson-McDaniel, S. Bernuzzi, C. M. Markakis, B. Brgmann, and W. Tichy, Phys. Rev. D 92, 124007 (2015).

[39] T. Dietrich, S. Bernuzzi, B. Brgmann, M. Ujevic, and W. Tichy, Phys. Rev. D 97, 064002 (2018).

[40] M. Ruiz, A. Tsokaros, V. Paschalidis, and S. L. Shapiro, Phys. Rev. D 99, 084032 (2019).

[41] E. R. Most, L. J. Papenfort, A. Tsokaros, and L. Rezzolla, Astrophys. J. 884, 40 (2019).

[42] A. Tsokaros, M. Ruiz, V. Paschalidis, S. L. Shapiro, and K. Ury, Phys. Rev. D 100, 024061 (2019).

[43] W. Kastaun, F. Galeazzi, D. Alic, L. Rezzolla, and J. A. Font, Phys. Rev. D 88, 021501 (2013).

[44] W. Kastaun and F. Galeazzi, Phys. Rev. D 91, 064027 (2015).

[45] W. Kastaun, R. Ciolfi, A. Endrizzi, and B. Giacomazzo, Phys. Rev. D 96, 043019 (2017).

[46] A. Bauswein, N. Stergioulas, and H.-T. Janka, Eur. Phys. J. A 52, 56 (2016).

[47] V. Paschalidis, W. E. East, F. Pretorius, and S. L. Shapiro, Phys. Rev. D 92, 121502 (2015).

[48] W. E. East, V. Paschalidis, F. Pretorius, and S. L. Shapiro, Phys. Rev. D 93, 024011 (2016).

[49] W.E. East, V. Paschalidis, and F. Pretorius, Classical Quantum Gravity 33, 244004 (2016).

[50] L. Lehner, S. L. Liebling, C. Palenzuela, and P. M. Motl, Phys. Rev. D 94, 043003 (2016).

[51] D. Radice, S. Bernuzzi, and C. D. Ott, Phys. Rev. D 94, 064011 (2016).

[52] D. Martynov et al., Phys. Rev. D 99, 102004 (2019).

[53] K. Kiuchi, K. Kyutoku, M. Shibata, and K. Taniguchi, Astrophys. J. 876, L31 (2019).

[54] B. P. Abbott et al. (LIGO Scientific and Virgo Collaborations), Phys. Rev. X 9, 011001 (2019).

[55] W. Tichy, Phys. Rev. D 84, 024041 (2011).

[56] W. Tichy, Phys. Rev. D 86, 064024 (2012).
[57] A. Tsokaros, K. Uryu, M. Ruiz, and S. L. Shapiro, Phys. Rev. D 98, 124019 (2018).

[58] W. E. East, F. Pretorius, and B. C. Stephens, Phys. Rev. D 85, 124010 (2012).

[59] W. E. East, F. Pretorius, and B. C. Stephens, Phys. Rev. D 85, 124009 (2012).

[60] T. W. Baumgarte, G. B. Cook, M. A. Scheel, S. L. Shapiro, and S. A. Teukolsky, Phys. Rev. D 57, 7299 (1998).

[61] A. Tsokaros, K. Ury, and L. Rezzolla, Phys. Rev. D 91, 104030 (2015).

[62] L. Engvik, G. Bao, M. Hjorth-Jensen, E. Osnes, and E. Ostgaard, Astrophys. J. 469, 794 (1996).

[63] J. S. Read, B. D. Lackey, B. J. Owen, and J. L. Friedman, Phys. Rev. D 79, 124032 (2009).

[64] J. S. Read, C. Markakis, M. Shibata, K. Uryū, J.D. E. Creighton, and J. L. Friedman, Phys. Rev. D 79, 124033 (2009).

[65] A. Bauswein, H.-T. Janka, and R. Oechslin, Phys. Rev. D 82, 084043 (2010).

[66] J. Barnes and D. Kasen, Astrophys. J. 775, 18 (2013).

[67] K. Kawaguchi, M. Shibata, and M. Tanaka, Astrophys. J. 865, L21 (2018).

[68] D. A. Coulter et al., Science 358, 1556 (2017).

[69] M. R. Drout et al., Science 358, 1570 (2017).

[70] B. J. Shappee et al., Science 358, 1574 (2017).

[71] M. M. Kasliwal et al., Science 358, 1559 (2017).

[72] M. Tanaka et al., Publ. Astron. Soc. Jpn. 69, 102 (2017),

[73] I. Arcavi et al., Nature (London) 551, 64 (2017).

[74] S. J. Smartt et al., Nature (London) 551, 75 (2017).

[75] M. Soares-Santos et al. (Dark Energy Camera GW-EM and DES Collaborations), Astrophys. J. 848, L16 (2017).

[76] M. Nicholl et al., Astrophys. J. 848, L18 (2017).

[77] P. S. Cowperthwaite et al., Astrophys. J. 848, L17 (2017).

[78] R. Fernández and B. D. Metzger, Mon. Not. R. Astron. Soc. 435, 502 (2013).

[79] B. D. Metzger and R. Fernández, Mon. Not. R. Astron. Soc. 441, 3444 (2014).

[80] A. Perego, S. Rosswog, R. M. Cabezon, O. Korobkin, R. Kappeli, A. Arcones, and M. Liebendörfer, Mon. Not. R. Astron. Soc. 443, 3134 (2014).

[81] O. Just, A. Bauswein, R. A. Pulpillo, S. Goriely, and H. T. Janka, Mon. Not. R. Astron. Soc. 448, 541 (2015).

[82] M.-R. Wu, R. Fernández, G. Martnez-Pinedo, and B. D. Metzger, Mon. Not. R. Astron. Soc. 463, 2323 (2016).

[83] J. Lippuner, R. Fernández, L. F. Roberts, F. Foucart, D. Kasen, B. D. Metzger, and C. D. Ott, Mon. Not. R. Astron. Soc. 472, 904 (2017).

[84] D. M. Siegel and B. D. Metzger, Phys. Rev. Lett. 119, 231102 (2017).

[85] A. Endrizzi, D. Logoteta, B. Giacomazzo, I. Bombaci, W. Kastaun, and R. Ciolfi, Phys. Rev. D 98, 043015 (2018).

[86] E. Nakar and T. Piran, Nature (London) 478, 82 (2011).

[87] P. Jaranowski, A. Królak, and B. F. Schutz, Phys. Rev. D 58, 063001 (1998).

[88] B. Sathyaprakash et al., Classical Quantum Gravity 29, 124013 (2012).

[89] H. Yang, V. Paschalidis, K. Yagi, L. Lehner, F. Pretorius, and N. Yunes, Phys. Rev. D 97, 024049 (2018).

[90] S. Bose, K. Chakravarti, L. Rezzolla, B. S. Sathyaprakash, and K. Takami, Phys. Rev. Lett. 120, 031102 (2018). 
[91] W. Kastaun, R. Ciolfi, and B. Giacomazzo, Phys. Rev. D 94, 044060 (2016).

[92] A. Endrizzi, R. Ciolfi, B. Giacomazzo, W. Kastaun, and T. Kawamura, Classical Quantum Gravity 33, 164001 (2016).

[93] M. Hanauske, K. Takami, L. Bovard, L. Rezzolla, J. A. Font, F. Galeazzi, and H. Stöcker, Phys. Rev. D 96, 043004 (2017).

[94] M. Hanauske, K. Takami, L. Bovard, L. Rezzolla, J. A. Font, F. Galeazzi, and H. Stöcker, Phys. Rev. D 96, 043004 (2017).

[95] R. Ciolfi, W. Kastaun, B. Giacomazzo, A. Endrizzi, D. M. Siegel, and R. Perna, Phys. Rev. D 95, 063016 (2017).

[96] R. Ciolfi, W. Kastaun, J. V. Kalinani, and B. Giacomazzo, Phys. Rev. D 100, 023005 (2019).
[97] K. Hotokezaka, K. Kiuchi, K. Kyutoku, H. Okawa, Y.-i. Sekiguchi, M. Shibata, and K. Taniguchi, Phys. Rev. D 87, 024001 (2013).

[98] Y. Sekiguchi, K. Kiuchi, K. Kyutoku, M. Shibata, and K. Taniguchi, Phys. Rev. D 93, 124046 (2016).

[99] D. Radice, F. Galeazzi, J. Lippuner, L. F. Roberts, C. D. Ott, and L. Rezzolla, Mon. Not. R. Astron. Soc. 460, 3255 (2016).

[100] L. Baiotti and L. Rezzolla, Rep. Prog. Phys. 80, 096901 (2017).

[101] V. Paschalidis and N. Stergioulas, Living Rev. Relativity 20, 7 (2017).

[102] D. Kasen, R. Fernández, and B. Metzger, Mon. Not. R. Astron. Soc. 450, 1777 (2015).

[103] R. Fernández and B. D. Metzger, Annu. Rev. Nucl. Part. Sci. 66, 23 (2016). 